\title{
Co-occurrence of Northern and Southern Hemisphere Blocks as Partially Synchronized Chaos
}

\author{
Gregory S. Duane,* Peter J. Webster, And Jeffrey B. Weiss \\ Program in Atmospheric and Oceanic Sciences, University of Colorado, Boulder, Colorado
}

(Manuscript received 29 April 1998, in final form 18 December 1998)

\section{ABSTRACT}

\begin{abstract}
Teleconnections between the midlatitudes of the Northern and Southern Hemispheres are diagnosed in National Centers for Environmental Prediction-National Center for Atmospheric Research reanalysis data and separately in European Centre for Medium-Range Weather Forecasts reanalysis data. The teleconnections are manifested as a small but significant tendency for blocking to occur simultaneously in the two hemispheres, though at different longitudes and different relative latitudes, during boreal winters over the period 1979-94 in both datasets.

One way to explain the correlations between blocking events is as an instance of synchronized chaos, the tendency of some coupled chaotic systems to synchronize, permanently or intermittently, regardless of initial conditions. As the coupling is weakened, the systems no longer synchronize completely, but small correlations between the states of the coupled systems are observed instead. In previous work, such behavior was observed in an idealized coupled-hemisphere model constructed from a midlatitude model due to de Swart, which extended the earlier Charney-DeVore spectral truncation of the barotropic vorticity equation by including a few extra modes. The direct coupling of the two midlatitude systems in the coupledhemisphere model represented the exchange of Rossby waves through the upper-tropospheric "westerly ducts" in the Tropics.

Significant correlations are found between blocking events, which are chaotically timed in each hemisphere considered singly, even without several of the idealizations used in the previous study. In a model modified to include an extended tropical region, the correlations are little affected by attenuation and phase shift of the Rossby waves that couple the two midlatitude systems. Variations in the relative longitudes of topographic features in the two hemispheres leave significant correlations or anticorrelations. The annual cycle, which imposes directionality on the coupling, since the Northern Hemisphere is more strongly forced than the Southern Hemisphere at the times when the hemispheres are coupled, increases the correlations slightly. A two-hemisphere model constructed from a higher-order (wavenumber 3 ) truncation of the barotropic vorticity equation exhibits regime transitions between blocked and zonal flow at a more realistic rate in each hemisphere but still exhibits interhemispheric correlations.
\end{abstract}

\section{Introduction and background}

Since the inception of the modern concept of deterministic chaos, considerable attention has been given to the proposition that large-scale atmospheric circulation might be governed by low-order chaotic dynamics. Low-order models of the midlatitude system, based on spectral truncations of the barotropic vorticity equation, have been proposed by Legras and Ghil (1985) and by de Swart $(1988,1989)$. Even more familiar is the use

\footnotetext{
* Current affiliation: National Center for Atmospheric Research Boulder, Colorado.
}

Corresponding author address: Gregory S. Duane, Advanced Study Program, National Center for Atmospheric Research, P.O. Box 3000, Boulder, CO, 80307-3000.

E-mail: gduane@ucar.edu of low-order chaos (Jin et al. 1994; Tziperman et al. $1995)$ to describe the irregularity of the El Niño-Southern Oscillation (ENSO) cycle found in operational models of the tropical coupled atmosphere-ocean system (Zebiak and Cane 1987). Recently, convincing evidence was presented that the aperiodicity in the Cane-Zebiak model of the ENSO cycle is indeed due to low-order chaos: periodicity can be restored by controlled feedback to a single degree-of-freedom in the model (Tziperman et al. 1997).

Yet a model of the global circulation as a single loworder system does not seem possible. A more modest goal, following a suggestion of Lorenz (1991), is to understand the emergent properties of a collection of loosely coupled chaotic systems, each describing a semiautonomous component of the global system. The coupling of the tropical and midlatitude systems, or of the monsoon and ENSO systems, may support descriptions of this type. 
A paradigm that may be relevant to the behavior of coupled atmospheric subsystems is that of synchronized chaos. Identical or similar chaotic systems can fall into synchronized motion along their strange attractors in a variety of unidirectional (Pecora and Carroll 1990; Kocarev et al. 1997) or bidirectional (Afraimovich et al. 1986; Fujisaka and Yamada 1983) coupling schemes. The tendency toward synchronization, at first surprising because of the sensitive dependence of each system on values of dynamical variables that are not shared, is possible because those variables that are shared capture the "chaotic" behavior of each system, so that the "subLyapunov" exponents, which describe the divergence of phase-space trajectories emanating from nearby points that differ only in the values of the unshared variables, are still negative (Pecora and Carroll 1990). As the coupling is weakened, synchronization degrades through on-off intermittency, that is, through bursts of desynchronization, timed chaotically, amidst otherwise synchronous behavior (Afraimovich et al. 1986).

Atmospheric teleconnections, as conceived originally by Walker (1924), imply a form of synchronized chaos in the context of Lorenz's (1991) view of the atmosphere as a collection of coupled chaotic subsystems. Walker, in his search for predictors of the Indian monsoon, postulated relationships between meteorological parameters at remote points on the globe, without knowledge of specific dynamical mechanisms for such connections, which were only elucidated much later (Bjerknes 1969; Hoskins and Karoly 1981; Webster 1981, 1982). In Walker's view, hope for monsoon prediction lay more with an understanding of such relationships than with an understanding of the regional dynamics of the monsoon system itself. Viewed in terms of the modern concept of synchronized chaos, each chaotic climate subsystem, such as the monsoon system, is unpredictable; yet, a teleconnection between two such subsystems is defined by correlations between their gross states. It is now known that strong monsoons, for instance, show a statistical tendency to occur in La Niña years and weak monsoons in El Niño years (Yasunari 1990; Webster and Yang 1992), though neither the monsoon nor ENSO is predictable more than a year in advance. But, while synchronized chaos has been investigated extensively in low-order systems, synchronization in naturally occurring or spatially extended systems, governed by partial, rather than ordinary differential equations, is relatively new (Kocarev et al. 1997; Sushchik 1996; Duane 1997).

By describing the large-scale atmospheric circulation in terms that allow for the possibility of synchronized chaos, we may gain insight regarding known teleconnection patterns and enable the prediction of new ones. For subsystems that are not identical, one must define a notion of corresponding states to define synchronization, and the correspondence may not be apparent a priori. Though there are algorithms to determine whether the trajectories of two coupled systems exhibit syn- chronization in this more general sense (Rulkov et al. 1995), the task of determining whether a correspondence between states exists and defining it is expectedly greater the greater the difference between the systems. The difficulty is exacerbated for spatially extended systems. We therefore begin by considering teleconnections between similar systems, where the similarity arises from an overall symmetry of the combined systems, which also makes the corresponding states obvious. Such a situation exists in the case of teleconnections between the two midlatitude systems because of reflection symmetry about the equator. The teleconnections are mediated by the exchange of Rossby waves through the tropical "westerly ducts" (Webster and Holton 1982), which interrupt the band of upper-tropospheric tropical easterlies.

Duane (1997) recently showed the existence of such teleconnections in a highly truncated (wavenumber 2) barotropic model of the interaction between the hemispheres constructed from $\beta$-plane models of the two midlatitude systems (de Swart 1988, 1989), assuming idealized geometry with a tropical easterly barrier that has no latitudinal extent, and ignoring the annual cycle. While the de Swart model requires unrealistically large thermal forcing to avoid stable fixed points, the interhemispheric teleconnections found in the two-hemisphere model would also be expected in a more realistic model based on spherical geometry. The teleconnections are manifested as a small but significant tendency for the two midlatitude systems to both exhibit blocked flow or to both exhibit zonal flow at the same instant of time.

It is shown here that a diagnostic used to define blocked flow in observed data in the Northern Hemisphere can be readily generalized for use in the Southern Hemisphere and is especially suited for computing cooccurrence statistics. Results indicate a correlation between blocking events, as predicted by the wavenumber 2 model. We then show that the correlations in this highly truncated model persist if we consider phase shifts and attenuation due to the finite latitudinal extent of the Tropics and that correlations are also found in a model that includes some higher modes in the midlatitudes. The specific form of the global topography used in the highly truncated model can also be relaxed. For a general longitudinal positioning of topographic features in the Southern Hemisphere, relative to those in the north, correlations or anticorrelations occur in the model. Finally, the annual cycle is shown to reinforce the correlations observed in the simple model, apparently by imposing a directionality on the coupling, with the summer hemisphere driven by the more strongly forced winter hemisphere to a slight degree.

\section{Southern Hemisphere blocking}

Teleconnections between the midlatitude regions of the two hemispheres are defined here in terms of correlations between the gross regimes simultaneously oc- 
cupied by the two midlatitude systems. The description of weather as a dichotomous system often appears natural, and correlations between regimes may give a first indication of correlations between meteorological parameters. It has been suggested by Palmer (1993), for instance, that the midlatitudes might be compared to a Lorenz system, with the two halves of the butterflyshaped attractor corresponding to zonal and blocked flow, respectively. The effect of the tropical-extratropical teleconnection, in this metaphorical view, is then to bias the Lorenz-system attractor so that the system spends more time on one-half of the butterfly than the other, depending on conditions in the Tropics that change over a longer timescale.

Atmospheric regimes are expectedly more difficult to define than the regimes of the symmetrical Lorenz system. While some earlier studies of hemispheric flow regimes (e.g., Webster and Keller 1974) were based on global diagnostics applied to the entire midlatitude flow to define an index cycle, this approach has generally been replaced by definitions of blocked states based on the presence of actual blocks at well-defined locations. Blocks have been defined differently by different authors (Rex 1950; Treidl et al. 1981; van Loon 1956; Wright 1974; Charney and DeVore 1979; Tibaldi and Molteni 1990). Since relatively little work has been done on Southern Hemisphere blocking, either theoretically or observationally, some care is devoted in this discussion to an appropriate definition of the blocked flow regime in the Southern Hemisphere. An early operational definition of blocking in the Northern Hemisphere was given by Rex (1950), who labeled a given circulation pattern as blocked if and only if the 500-mb westerly current splits into two branches, each of which transports an appreciable mass in a split pattern extending over at least $45^{\circ}$ of longitude and persisting continuously for at least 10 days, causing a sharp transition between an upstream zonal-type flow and a downstream meridional-type flow. Treidl (1981) added a requirement that there be a surface high, shortened the required duration to 5 days, and excluded blocks extending south of $30^{\circ} \mathrm{N}$ (considering only Northern Hemisphere blocks). Blocking events thus defined were found to occur primarily over the Atlantic or Pacific Oceans at an average latitude of $56^{\circ} \mathrm{N}$.

A less arbitrary, more easily programmed definition of blocking was given by Tibaldi and Molteni (1990), who defined a block in the Northern Hemisphere as a coarse-resolution local maximum of 5-day-averaged 500 -mb height on a given meridian, with the requirement that the gradient of height on the northern side of the block exceed a given threshold so as to exclude artifacts arising from large southward displacement of the midlatitude westerly jet. Formally, the Tibaldi-Molteni diagnostic is defined in terms of the geopotential height gradients for each longitude $\lambda$, at each instant of time $t$ :

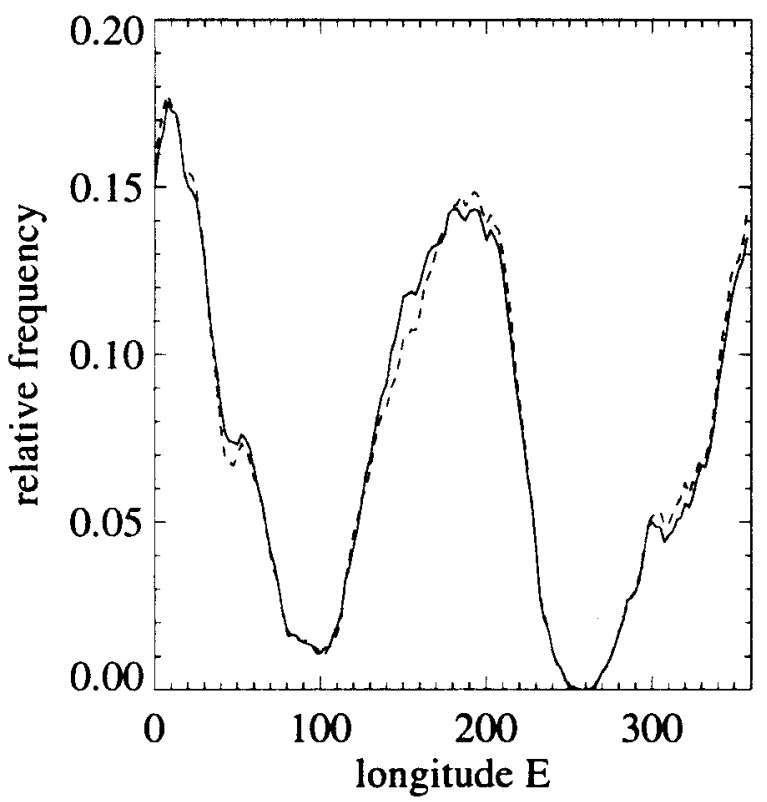

FIG. 1. Fraction of all analysis times at which the Northern Hemisphere circulation is blocked at given longitude, according to the Tibaldi-Molteni diagnostic, in the 1979-94 NCEP-NCAR data (solid line), and in the 1979-93 ECMWF data (dashed line) during boreal winters. The resolution in both datasets is $2.5^{\circ}$ in both latitude and longitude.

$$
\begin{aligned}
& \operatorname{GHG} 1(\lambda, t)=\frac{z\left(\phi_{0}, \lambda, t\right)-z\left(\phi_{-}, \lambda, t\right)}{\phi_{0}-\phi_{-}} \\
& \operatorname{GHG} 2(\lambda, t)=\frac{z\left(\phi_{+}, \lambda, t\right)-z\left(\phi_{0}, \lambda, t\right)}{\phi_{+}-\phi_{0}},
\end{aligned}
$$

where $z$ is the 5-day-averaged geopotential height,

$$
\begin{aligned}
& \phi_{0}=60^{\circ} \mathrm{N}+\Delta \\
& \phi_{+}=\phi_{0}+20^{\circ}+\Delta \\
& \phi_{-}=\phi_{0}-20^{\circ}+\Delta,
\end{aligned}
$$

and

$$
\Delta=-5^{\circ}, 0^{\circ} \text {, or }+5^{\circ} \text {. }
$$

(The values $\Delta=-4^{\circ}, 0^{\circ}$, or $+4^{\circ}$ were originally used by Tibaldi and Molteni for an earlier dataset with a different grid interval.) The circulation is said to be blocked at longitude $\lambda$ and time $t$ if and only if

$$
\operatorname{GHG} 1(\lambda, t)>0 \text { and } \operatorname{GHG} 2(\lambda, t)<-10 \mathrm{~m} \mathrm{deg}^{-1}
$$

for at least one value of $\Delta$ in (3). In the Northern Hemisphere $\phi_{0}$ is chosen to be $60^{\circ} \mathrm{N}$, requiring blocks to be centered within $5^{\circ}$ of $60^{\circ} \mathrm{N}$. Blocking frequency computed with the diagnostic (4) for each longitude $\lambda$, using National Centers for Environmental Prediction-National Center for Atmospheric Research (NCEP-NCAR) data (Kalnay et al. 1996), is plotted in Fig. 1. The dashed line in the figure gives the frequencies computed using 
the European Centre for Medium-Range Weather Forecasts (ECMWF) dataset instead. Results for the two datasets agree well. Tibaldi and Molteni stressed that such definitions, not involving additional requirements of minimum duration and longitudinal extent, were particularly useful in "comparison mode," as in comparing analyses to forecasts or one longitude to another. Here, the Tibaldi-Molteni definition is used to study the cooccurrence of blocked states in the Northern and Southern Hemispheres.

Most observational work on Southern Hemisphere blocking stems from the work of van Loon (1956), who compiled statistics using a definition similar to Rex's (1950). Van Loon's definition, as modified by Wright (1974), differed from the early definitions of blocking in the Northern Hemisphere principally in that it was necessary to exclude the climatological subtropical high in the Southern Hemisphere and fast-moving high pressure centers. These early studies found three centers of blocking activity in the Southern Hemisphere, corresponding to the three major land masses (though they occur downstream of these land masses, unlike their Northern Hemisphere counterparts, which occur upstream of the two major land masses). Blocks were found to occur with frequency comparable to that of Northern Hemisphere blocks but are less intense, of shorter duration, and occur at more equatorward latitudes.

The Tibaldi-Molteni diagnostic can be extended to the Southern Hemisphere by replacing the latitudes in (2) by suitable southern latitudes. We choose $\phi_{0}=40^{\circ} \mathrm{S}$ to detect blocks formally centered at $35^{\circ}, 40^{\circ}$, or $45^{\circ} \mathrm{S}$, obtaining the longitudinal distribution of blocking shown in Fig. 2, where attention is restricted to the boreal winter months, a period of relatively frequent blocking in both hemispheres. It was found that higher southern latitudes exhibited no significant blocking, according to this diagnostic, as can be seen from the dotted line in Fig. 2, which represents the effect of including an additional value of $\Delta=+10^{\circ}$ in (3). The use of lower latitudes, on the other hand, leads to the confusion of tropical phenomena with midlatitude blocking events.

One might seek to lower a threshold in the diagnostic to detect Southern Hemisphere blocks since these have been reported to be less intense (Coughlan 1983) than Northern Hemisphere blocks. That Southern Hemisphere blocks are weaker is indeed confirmed in Fig. 3, displaying Hovmöller plots, for both hemispheres, of the quantity GHG1 (the maximum value over the three possible values of $\Delta$ ) for a typical boreal winter period at locations and times where both conditions in (4) are satisfied. (The GHG2 condition is less restrictive than the GHG1 condition and so is of less value as an indication of blocking intensity. In the Northern Hemisphere, the GHG2 condition is used only to distinguish between blocks and equatorward displacements of the midlatitude jet. In the Southern Hemisphere, the GHG2 condition is found to be satisfied everywhere that the

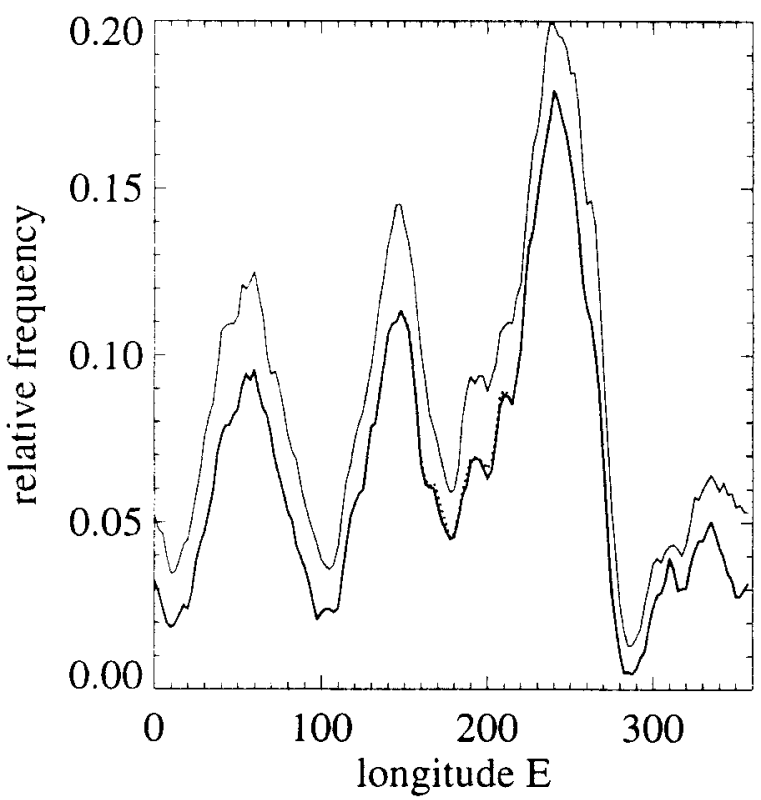

FIG. 2. Blocking frequencies as in Fig. 1 (NCEP-NCAR data only) but for the Southern Hemisphere (thick line), using the same blocking diagnostic, except applied at $40^{\circ} \mathrm{S}$ instead of $60^{\circ} \mathrm{N}$. Results are also shown for the same diagnostic modified to include an additional value of $\Delta=10^{\circ}$ in (3) (dotted line), to detect any blocking at higher southern latitudes, and using 3-day-averaged height data (thin solid line) instead of 5-day-averaged data.

GHG1 condition is satisfied.) However, the magnitude of GHG1 plays no role in the diagnostic itself. Nor is there another apparent way to modify the diagnostic to detect weaker blocking events. Changing the length scale over which the gradients are computed (from $20^{\circ}$ to $10^{\circ}$ or $5^{\circ}$ ) was not found to be useful. To increase sensitivity to short-lived events, we examined results obtained with a 3-day average instead (thin line in Fig. 2) and found a small increase in blocking frequency but in almost exact proportion to the original frequency at each longitude. We therefore maintain the 5-day average in our definition for uniformity with the Northern Hemisphere definition.

Although van Loon (1956) and Wright (1974) imposed restrictions on the rate of zonal progression of a moving block, such restrictions are not needed for the purpose of comparing analyses to forecasts or comparing "blocking" frequencies at different longitudes. Similarly, they will not be needed for the purpose of comparing blocking behavior in the two hemispheres. Of greater importance are the attempts of both these authors to distinguish, in their definitions, between blocking events and the climatological subtropical high, which is extracted from the ECMWF data in Fig. 4. (In the Northern Hemisphere, where blocks occur at higher latitudes, the distinction is obvious.) As can be seen in the figure, the subtropical high pressure center off the western coast of South America (about $35^{\circ} \mathrm{S}$ ) is bigger and a bit more southerly than the other high pressure centers. If we 
a)

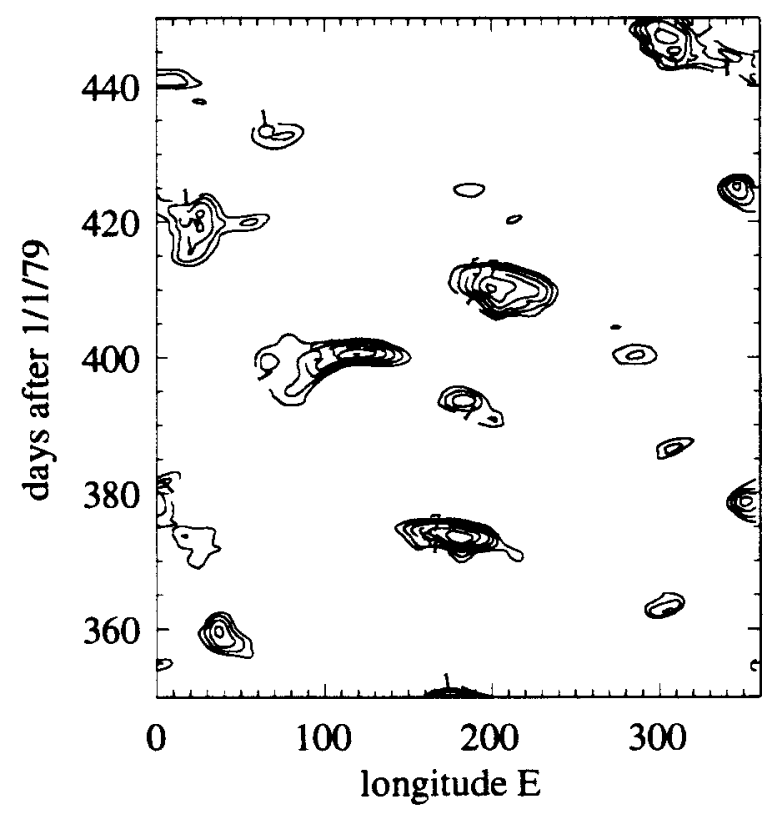

b)

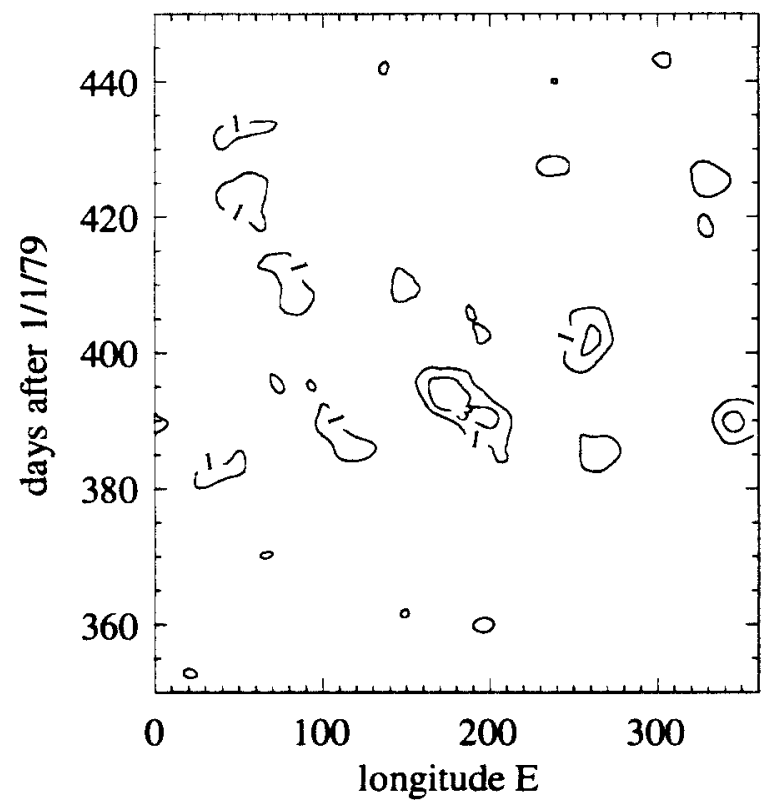

FIG. 3. Hovmöller plots of blocking intensity, represented by the maximum value of GHG1 in (1) over the three values of $\Delta$ at each longitude and time in a boreal winter period in the second year of the NCEP-NCAR data, for (a) the Northern Hemisphere at $60^{\circ} \mathrm{N}$ and (b) the Southern Hemisphere at $40^{\circ} \mathrm{S}$. Only positive values of GHG1, at locations for which GHG $2<-10 \mathrm{~m}^{\text {degree }}{ }^{-1}$, corresponding to blocked states, are plotted. The contour interval is $2 \mathrm{~m}$ degree $^{-1}$. adopt Wright's (1974) requirement that part of the block be at least $7^{\circ}$ south of these high pressure centers and interpret the region of the block as extending $\Delta=5^{\circ}$ north and south of the formal center, we are led to exclude the value $\Delta=-5^{\circ}$, corresponding to a formal center at $35^{\circ}$, from the set (3), in defining blocking in this region of the South Pacific. Taking this region $\mathcal{R} \equiv$ $\left\{\lambda \mid 160^{\circ} \mathrm{W}<\lambda<60^{\circ} \mathrm{W}\right\}$ as a rough estimate, we replace (3) by

$$
\Delta= \begin{cases}0^{\circ} \text { or } 5^{\circ} & \text { if } \lambda \in \mathcal{R} \\ -5^{\circ}, 0^{\circ}, \text { or } 5^{\circ} & \text { otherwise. }\end{cases}
$$

The longitudinal distribution of blocking, according to the Tibaldi-Molteni definition, thus modified, is shown in Fig. 5, for the NCEP-NCAR and ECMWF reanalysis datasets. The large peak at $120^{\circ} \mathrm{W}$ in Fig. 2, corresponding to the climatological subtropical high, has been eliminated in Fig. 5, though there is a small vestige of it in the case of the ECMWF data. The difference between the two distributions in Fig. 5 is notably larger than for the distributions in the Northern Hemisphere, shown in Fig. 1, presumably because of the relative scarcity of observations and hence greater sensitivity to differences between forecast models used in the Southern Hemisphere. The slight southward displacement of the climatological high pressure center off the coast of South America, as compared to the other two centers, was in fact not detected in the NCEP-NCAR dataset. Nevertheless, we regard the displacement of this center in the ECMWF data as evidence that the large peak in Fig. 2 is due to the subtropical high and not to blocking.

For both distributions shown in Fig. 5, the peaks correspond roughly to the three major areas of Southern Hemisphere blocking identified by van Loon (1956). Error in the plots, which is primarily due to error in forecast models rather than instrument error, can be inferred from the difference between the ECMWF and NCEP-NCAR distributions, implying that only the broad structure of the peaks is meaningful. The peak centered at $50^{\circ} \mathrm{E}$ agrees well with van Loon's Indian Ocean center. The double peak between about $125^{\circ} \mathrm{E}$ and $160^{\circ} \mathrm{W}$ similarly agrees with van Loon's AustralianPacific region. The broad peak in our figure centered about $30^{\circ} \mathrm{W}$ is displaced by about $20^{\circ}$ from van Loon's Atlantic center and is smaller in magnitude, but the correspondence is clear. Given that no satellite data were used in van Loon's analysis, the agreement between our distribution and his is striking and tends to validate our choice of blocking diagnostic.

\section{Co-occurrence of Northern and Southern Hemisphere blocks in observed data}

Any relationships between the two possible regimes (zonal or blocked) occupied simultaneously by the two hemispheres can be gleaned naively from the rates of occurrence of the four possible combinations. We ex- 


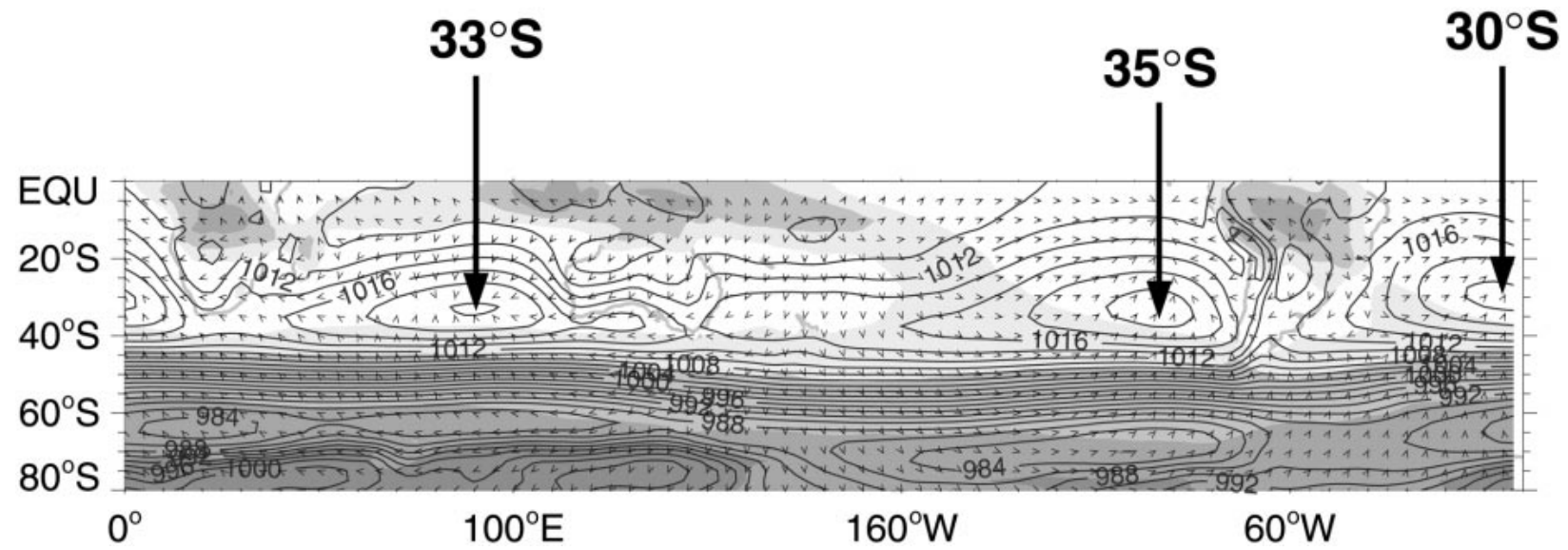

FIG. 4. Climatological surface pressure in ECMWF data for 1979-93 boreal winters. Latitudes of the subtropical high pressure centers are indicated. (Other climatological data are also indicated: arrows represent wind speed and shading represents outgoing longwave radiation.)

amine the cooccurrence statistics for these four possible regime pairs in the NCEP-NCAR data, expressed as a matrix of joint probabilities $P_{i, j}$ [where $i, j=z$ (zonal) or $b$ (blocked)] in Table 1 . The probability $P_{i, j}$ is the fraction of all analysis times, during boreal winters, at which the Northern Hemisphere is in regime $i$ and the Southern Hemisphere is in regime $j$. Also shown are the corresponding probability values $P_{i, j}^{\text {ind }}$ in the case where the two hemispheres individually have the same statistics but are assumed independent, that is,

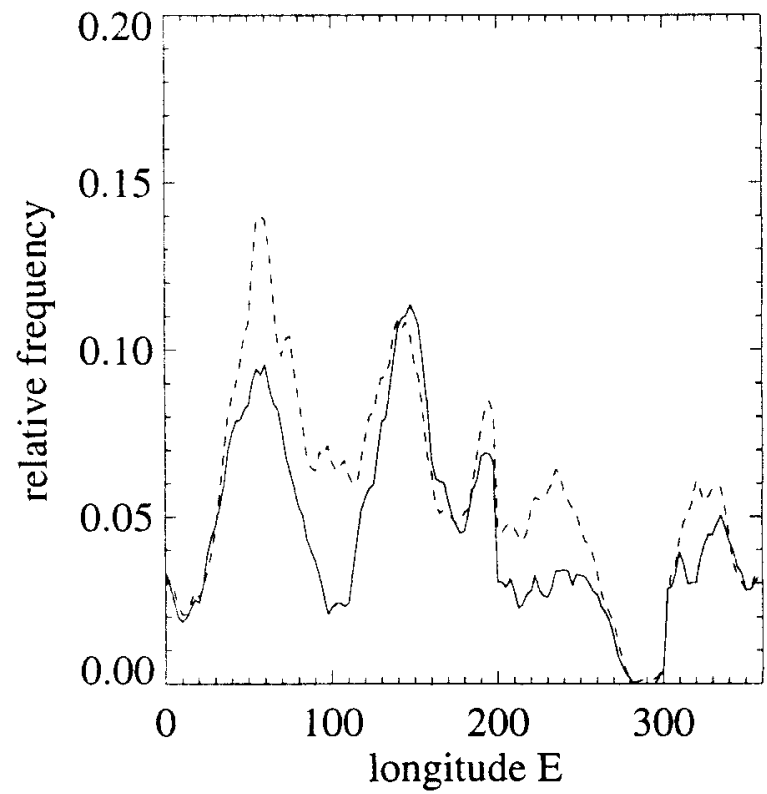

FIG. 5. Blocking frequencies for boreal winters in Fig. 2, except with the modified diagnostic in (4) and (5) that excludes too close to the climatological subtropical high. Results are shown for the NCEPNCAR data (solid line) and for the ECMWF data (dashed line).

$$
P_{i, j}^{\text {ind }}=\left(\sum_{r=z, b} P_{i, r}\right)\left(\sum_{s=z, b} P_{s, j}\right)
$$

The diagonal elements of the matrix $\mathbf{P}$ are larger than those of the matrix Pind, while the off-diagonal elements are smaller. This configuration indicates correlation. The standard correlation between the two binary-valued random variables $Q$ and $R$, which label the regimes of the two subsystems, is defined as $C \equiv\langle(Q-\langle Q\rangle)(R-\langle R\rangle)\rangle /$ $\left(\sigma_{Q} \sigma_{R}\right)$, where $Q=1(R=1)$ when the Northern Hemisphere (Southern Hemisphere) is blocked and $Q=0(R$ $=0)$ otherwise. $(\langle\rangle$ denotes a time average. $)$ The correlation can also be expressed in terms of the joint probabilities as

$$
C \equiv \frac{P_{b b}-P_{b b}^{\text {ind }}}{\sqrt{\sum_{r=z, b} P_{z, r} \sum_{r=z, b} P_{b, r} \sum_{s=z, b} P_{s, z} \sum_{s=z, b} P_{s, b}}} .
$$

A significantly positive correlation value is shown in

TABLE 1. (a) Relative frequencies of cooccurrence of regime pairs in NCEP-NCAR data for boreal winters in the period 1979-94, represented as joint probabilities $P_{i, j}$. Regimes are defined using the diagnostic in (4) and (5). (b) The joint probabilities $P_{i, j}^{\text {ind }}$ for independent subsystems, each with the same probability of occupying either regime as in (a), i.e., $P_{i, j}^{\text {ind }}=\left(\sum_{j=z, b} P_{i, j}\right)\left(\sum_{i=z b} P_{i, j}\right)$. The correlation $C$ is defined in (7). Error bars on $C$ were computed as twice the standard error of the mean in a partitioned dataset. The value $p$ is the probability that the correlation value $C$ would be obtained for uncorrelated time series of the same length, assuming that the values $C$ are chisquared distributed with a decorrelation time of 15 days.

\begin{tabular}{lccccc}
\hline \hline a) & \multicolumn{2}{c}{$\mathbf{P}$} & & \multicolumn{2}{c}{ (b) } \\
\cline { 2 - 3 } \cline { 5 - 5 } & N zonal & N blocked & & N zonal & N blocked \\
\hline S zonal & 0.21 & 0.23 & & 0.16 & 0.27 \\
S blocked & 0.16 & 0.40 & & 0.20 & 0.36 \\
& & $C=0.20 \pm 0.14$ & & $p=0.05$ \\
\hline
\end{tabular}


TABLE 2. Co-occurrence matrices, correlation coefficient, and $p$ value as in Table 1 but for the ECMWF 1979-93 dataset.

\begin{tabular}{lccccc}
\hline \hline (a) & \multicolumn{2}{c}{$\mathbf{P}$} & & \multicolumn{2}{c}{ (b) } \\
\cline { 2 - 3 } \cline { 5 - 6 } & N zonal & N blocked & & N zonal & N blocked \\
\hline S zonal & 0.19 & 0.19 & & 0.14 & 0.24 \\
S blocked & 0.17 & 0.45 & & 0.22 & 0.40 \\
& & $C=0.23$ & & $p=0.03$ \\
\hline
\end{tabular}

Table 1. Interestingly, this correlation value was unchanged when the simpler diagnostic that does not adjust for the presence of the climatological subtropical high, was used to define Southern Hemisphere blocking (though the individual $P_{i j}$ values did change). Since there is no reason to expect small fluctuations in the subtropical high that might be mistaken for blocking events to correlate with synoptic regimes in the opposite hemisphere, this result also tends to validate our somewhat crude method of distinguishing between the subtropical high and blocking events.

The 2- $\sigma$ error shown in the table was obtained by partitioning the data into five subsets and computing the standard error of the mean. As an additional significance test, we computed the variance about zero correlation in collections of uncorrelated datasets created artificially by shifting the time series in one of the hemispheres, giving a 2- $\sigma$ error equal to or lower than that shown in the table. As a third significance test, we assumed that the correlation values $C$ are chi-squared distributed, that is, $\chi^{2}=n C^{2}$, with $n$ denoting the number of decorrelation intervals in the entire time series. The probability $p$, that any value $\chi^{\prime}$ in the distribution is such that $\chi^{\prime}$ $\geq \chi$, is the probability that two randomly chosen time series of uncorrelated data of equal length will exhibit a correlation that exceeds $\mathcal{C}$. A $p$ value of 0.05 or less, as shown in the table for an assumed maximum decorrelation time of 15 days, indicates significant correlation.

A similar interhemispheric correlation is found for the ECMWF data, analyzed in Table 2, despite the differences between the two datasets seen in Fig. 5. Lagged correlations are plotted for both datasets in Fig. 6. The largest correlations are simultaneous, not delayed, although there is some asymmetry with respect to time ordering in the lagged correlations, which are smaller and marginally significant.

Comparing the boreal winter statistics, on the other hand, to statistics for the entire period 1979-94, we see that the correlation $C$ for the full-year NCEP-NCAR data shown in Table 3 is reduced. Taking the traditional view of teleconnections as mediated by the exchange of Rossby waves (e.g., Hoskins and Karoly 1981), the enhanced correlations during winters can perhaps be understood. Rossby waves between the hemispheres can propagate through the Tropics only at longitudes where the upper tropospheric winds are westerly, as illustrated in the simulation in Fig. 7 (Webster and Holton 1982),

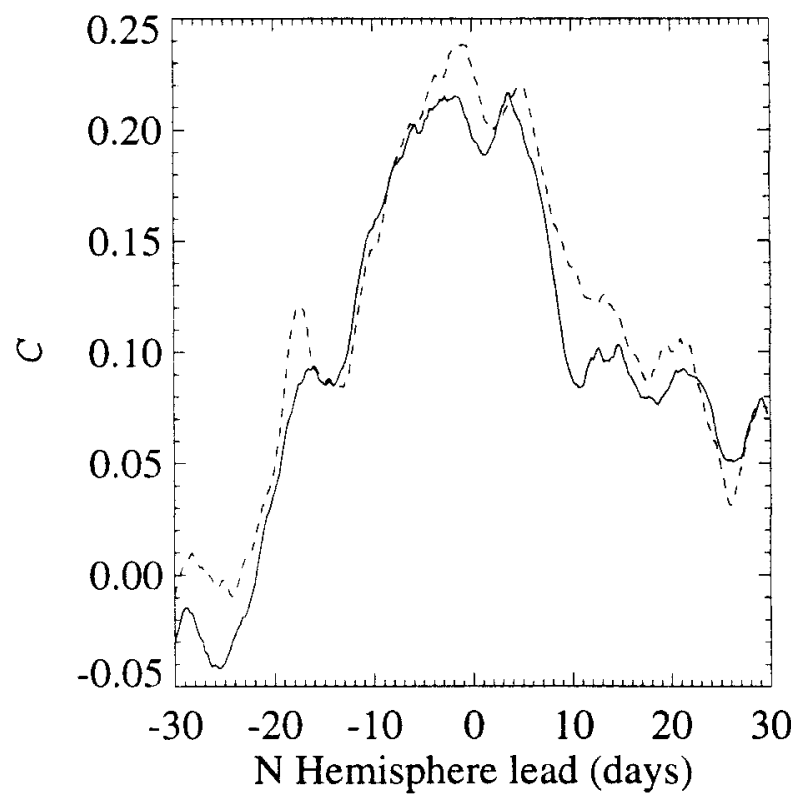

FIG. 6. Lagged correlation values $\mathcal{C}$ defined as in Table 1 but for the Northern Hemisphere time series of regime labels shifted, relative to the Southern Hemisphere time series, by a number of days indicated on the horizontal axis. (Negative values represent Southern Hemisphere lead.) Correlations are shown for NCEP-NCAR data (solid line) and ECMWF data (dashed line) for boreal winters.

for reasons reviewed in the next section. As shown in the Hovmöller plot of upper tropospheric winds in Fig. 8 , such regions, the westerly ducts, open and close seasonally. Strong westerlies, which allow the least attenuated transmission of Rossby waves, exist only during boreal winters. If the correlations linking blocking activity in the two hemispheres are due to the exchange of Rossby waves, at least in part, then the correlation values should be greater during the winter months.

One might also ask whether the correlations are enhanced if we restrict attention to a specific band of longitudes, in the vicinity of the westerly ducts. Statistics for the Pacific region are displayed in Table 4. It is seen that blocking activity in the North Pacific is no more likely to be accompanied by blocking in the South Pacific than it is to be accompanied by blocking anywhere else in the Southern Hemisphere. The Rossby wave signal through the westerly ducts, if this is indeed the cause of the correlations, would appear to affect the circulation of the entire opposite hemisphere.

TABLE 3. Relative frequencies of co-occurrence of regime pairs in NCEP-NCAR data as in Table 1 but for full-year statistics.

\begin{tabular}{lccccc}
\hline \hline (a) & \multicolumn{2}{c}{$\mathbf{P}$} & & \multicolumn{2}{c}{ Pind } \\
\cline { 2 - 3 } \cline { 5 - 6 } & N zonal & N blocked & & N zonal & N blocked \\
\hline S zonal & 0.33 & 0.44 & & 0.32 & 0.46 \\
S blocked & 0.08 & 0.15 & & 0.09 & 0.13 \\
& & $C=0.07$ & & $p=0.50$ \\
\hline
\end{tabular}



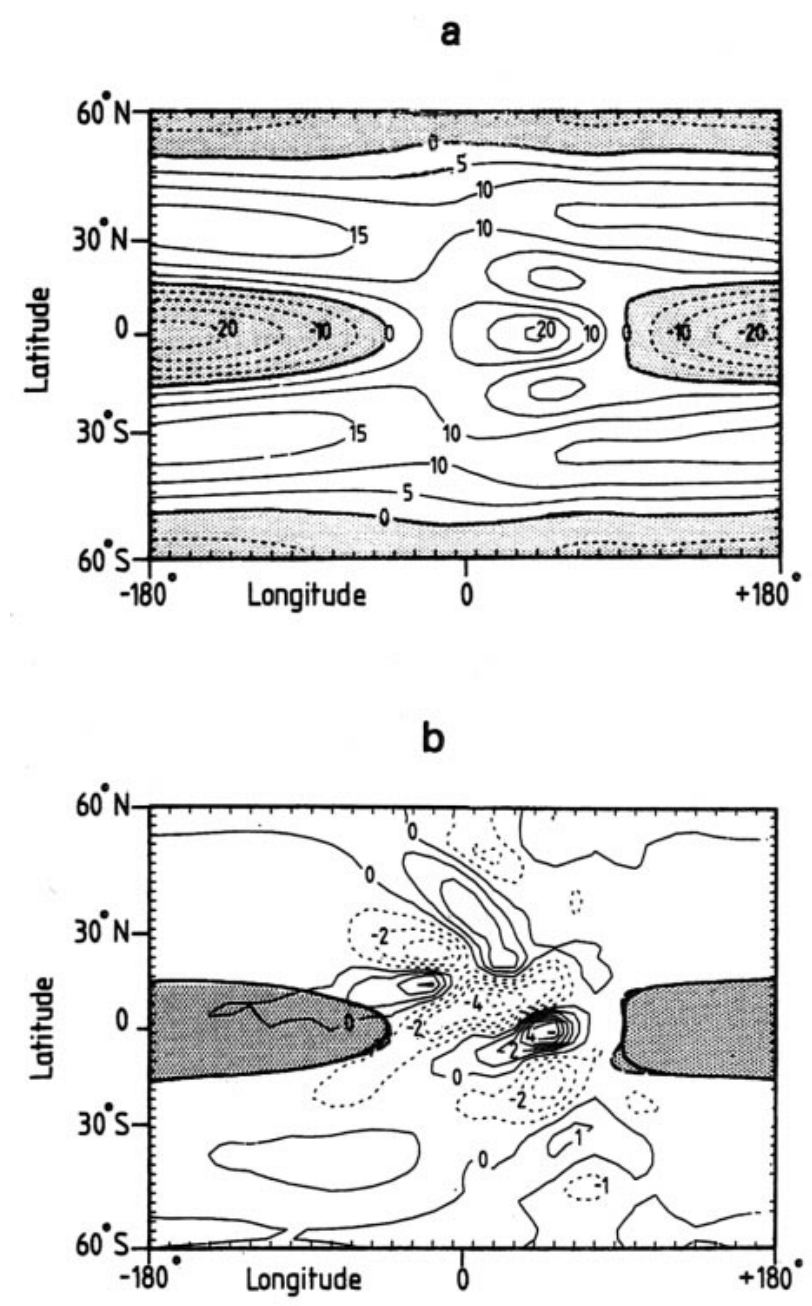

FIG. 7. Rossby wave penetration of the tropical westerly ducts in a high-resolution numerical model of shallow-fluid dynamics: (a) Contours of a prescribed basic-state zonal wind field $\bar{u}$ (in $\mathrm{m} \mathrm{s}^{-1}$ ), intended to represent climatological conditions in the Tropics. Regions of easterly wind $\bar{u}<0$ are shaded. (b) Contours of perturbation zonal wind $u$ at $t=100$ days, with basic state as in (a), after initialization by a localized perturbation at $20^{\circ} \mathrm{N}$ at $t=0$ [reprinted by permission from Webster and Holton (1982)].

\section{The synchronized chaos model}

\section{a. Low-order models of the separate midlatitude systems}

In this section, we sketch the derivation of Duane's (1997) model of the coupled hemispheres so that we may augment it to capture several features of the real atmosphere that were ignored in the previous study.

Regime transitions between blocked and zonal flow in each hemisphere can be conceptualized as the result of low-order chaos. A low-order chaotic model of the midlatitudes in one hemisphere, due to de Swart (1988, 1989), appears to capture the qualitative features of the large-scale circulation. The model is constructed from the barotropic vorticity equation:

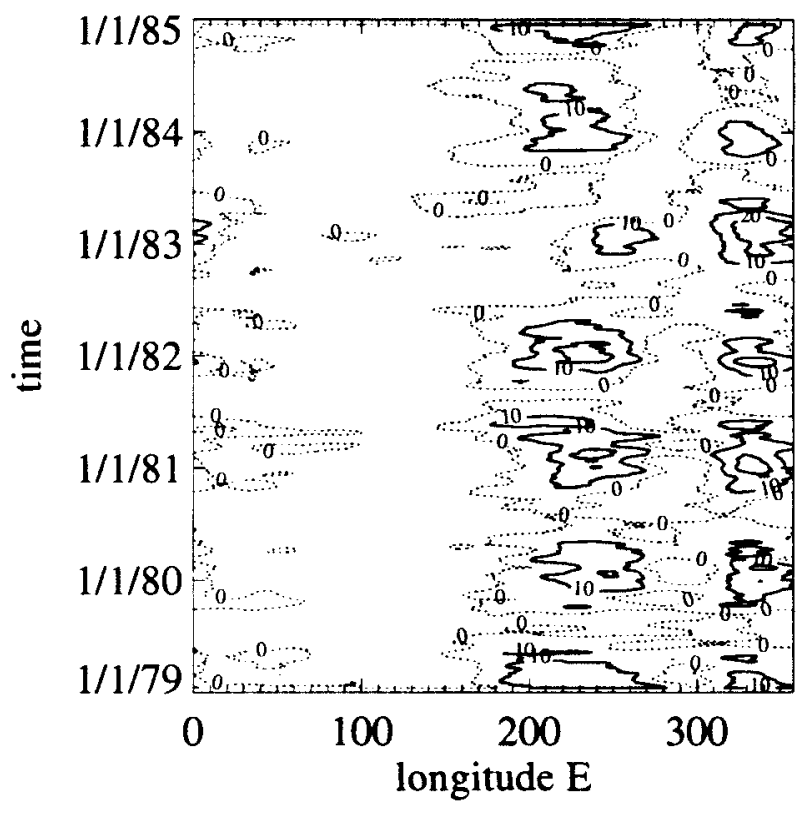

FIG. 8. Contour plot of the zonal component of the observed uppertropospheric $(200 \mathrm{mb})$ tropical wind $u$ (in $\left.\mathrm{m} \mathrm{s}^{-1}\right)$, which defines the westerly ducts, vs longitude and time. The tropical wind is defined as the average of the wind between $10^{\circ} \mathrm{N}$ and $10^{\circ} \mathrm{S}$, also averaged over 30 days. The contour interval is $10 \mathrm{~m} \mathrm{~s}^{-1}$. Solid contours represent positive (westerly) values of the wind. Dotted contours represent $u=0$ (computed from NCEP-NCAR reanalysis data).

$$
\begin{aligned}
& \frac{\partial\left(\nabla^{2} \Psi\right)}{\partial t}+J\left(\Psi, \nabla^{2} \Psi+f\right)+\gamma J(\Psi, h) \\
& \quad+\mu \nabla^{2}\left(\Psi-\Psi^{*}\right)=0,
\end{aligned}
$$

where $\Psi$ is the streamfunction, which gives the $x$ and $y$ components of horizontal velocity $u=-\partial \Psi / \partial y, v=$ $\partial \Psi / \partial x ; t$ is time; $f$ is twice the locally vertical component of the rotation vector, which defines the Coriolis force; $h$ is the bottom topographic height; $\gamma=f_{0} h_{0} / H$ is a coefficient of topographic forcing; $f_{0}$ is a typical value of $f ; H$ is the average height of the atmosphere, $h_{0}$ is a topographic height scale; and $\mu$ is a coefficient of bottom friction. The forcing streamfunction $\Psi^{*}$ represents the flow forced by the equator-to-pole temperature gradient, which would define an equilibrium state in the absence of topographic effects. The advection Jacobian $J$ is

TABLE 4. Relative frequencies of co-occurrence of regime pairs in NCEP-NCAR data as in Table 1 but only considering blocks in the Pacific region between $120^{\circ} \mathrm{E}$ and $150^{\circ} \mathrm{W}$. (Circulation patterns with blocks occurring at other longitudes are counted as "zonal.")

\begin{tabular}{lccccc}
\hline \hline (a) & \multicolumn{2}{c}{$\mathbf{P}$} & & \multicolumn{2}{c}{ (b) } \\
\cline { 2 - 3 } \cline { 5 - 6 } & N zonal & N blocked & & N zonal & N blocked \\
\hline S zonal & 0.45 & 0.21 & & 0.43 & 0.23 \\
S blocked & 0.20 & 0.14 & & 0.22 & 0.12 \\
& & $C=0.09$ & & $p=0.39$ \\
\hline
\end{tabular}




$$
J(A, B) \equiv \frac{\partial A}{\partial x} \frac{\partial B}{\partial y}-\frac{\partial A}{\partial y} \frac{\partial B}{\partial x} .
$$

The midlatitude system is represented as a narrow channel between two circles of latitude, of width $B$ (in the meridional, or $y$, dimension) and length $L$ (in the zonal, or $x$, dimension), with periodic boundary conditions in $x$. The conditions imposed at the northern and southern boundaries are that the meridional wind $v$ and the circulation $\int u d x$ vanish, that is,

$$
\begin{array}{rlrl}
\frac{\partial \Psi}{\partial x}=0 & y=0 & \text { or } \quad y=\pi b \\
\int \frac{\partial \Psi}{\partial y} d x=0 & y=0 & \text { or } \quad y=\pi b,
\end{array}
$$

where we have nondimensionalized $x$ and $y$ by defining a length scale $L / 2 \pi$ and we have introduced the nondimensional constant $b \equiv 2 B / L$. The channel is assumed to lie on a $\beta$ plane, with $\partial f / \partial y=\beta$. The time $t$ is nondimensionalized by defining a timescale equal to $10^{5}$ $\mathrm{s}=1.16$ days, giving a nondimensional $\beta$ of order unity.

A six-component spectral truncation of the barotropic vorticity equation (8) was shown by Charney and Devore (1979) to exhibit multiple equilibria corresponding to different weather regimes in the atmosphere. More recently, de Swart $(1988,1989)$ showed that a corresponding 10-component spectral truncation of (8) exhibits chaotic behavior with a strange attractor that gives vacillation between regimes. De Swart projected onto the basis $\Phi_{j}=\Phi_{j_{1}, j_{2}}$ for $j_{1}$ an integer and $j_{2}$ a positive integer:

$$
\begin{aligned}
& \Phi_{0, j_{2}}=\sqrt{2} \cos \left(j_{2} \frac{y}{b}\right) \\
& \Phi_{j_{1}, j_{2}}=\sqrt{2} e^{i j_{1} x} \sin \left(j_{2} \frac{y}{b}\right) .
\end{aligned}
$$

The corresponding eigenvalues $\lambda_{j}$, defined by $\nabla^{2} \Phi_{j}=$ $-\lambda_{j} \Phi_{j}$ [where $\left.j=\left(j_{1}, j_{2}\right)\right]$, are $\lambda_{j}=j_{1}^{2}+j_{2}^{2} / b^{2}$. The $j_{1}$ $=0$ modes consist of purely zonal flow $\left(v=-\partial \Phi_{0, j_{2}} /\right.$ $\partial x=0$ ), while the $\left|j_{1}\right|>0$ modes consist of Rossby waves that are traveling in $x$ and standing in $y$. That is, the sreamfunction $\Psi(x, y, t)=\Phi_{j_{1} j_{2}} e^{-i \omega_{j} t}\left(\left|j_{1}\right|>0\right)$, where

$$
\omega_{j}=\frac{-\beta j_{1}}{\lambda_{j}}=\frac{-\beta j_{1}}{j_{1}^{2}+\left(j_{2} / b\right)^{2}}
$$

is a Rossby wave that satisfies (8) with $h=\mu=\Psi^{*}$ $=0$.

The expansion of the streamfunction $\Psi$, the forcing "streamfunction" $\Psi^{*}$, and the topography $h$ in the basis (11) is defined by

$$
\left(\Psi, \Psi^{*}, h\right)=\sum_{j}\left(\psi_{j}, \psi_{j}^{*}, h_{j}\right) \Phi_{j} .
$$

The projection of the barotropic vorticity equation (8) onto the the eigenfunctions (11) is found to be
TABLE 5. The truncated basis that defines the de Swart $(1988,1989)$ model of the single-hemisphere midlatitude circulation, given by the streamfunction $\Psi=\sum_{i=1}^{10} \mathrm{x}_{i}(t) Y_{i}$.

\begin{tabular}{ll}
\hline \hline$\Upsilon_{1}=\sqrt{2} b \cos \frac{y}{b}$ & $\Upsilon_{2}=2 b \cos x \sin \frac{y}{b}$ \\
$\Upsilon_{3}=2 b \sin x \sin \frac{y}{b}$ & $\Upsilon_{4}=\sqrt{2} b \cos \frac{2 y}{b}$ \\
$\Upsilon_{5}=2 b \cos x \sin \frac{2 y}{b}$ & $\Upsilon_{6}=2 b \sin x \sin \frac{2 y}{b}$ \\
$\Upsilon_{7}=2 b \cos 2 x \sin \frac{y}{b}$ & $\Upsilon_{8}=2 b \sin 2 x \sin \frac{y}{b}$ \\
$\Upsilon_{9}=2 b \cos 2 x \sin \frac{2 y}{b}$ & $\Upsilon_{10}=2 b \sin 2 x \sin \frac{2 y}{b}$ \\
\hline
\end{tabular}

$$
\begin{aligned}
\lambda_{j} \dot{\psi}_{j}= & \frac{1}{2} \sum_{l, m} c_{j l m}\left(\lambda_{l}-\lambda_{m}\right) \psi_{l} \psi_{m}+\gamma \sum_{l, m} c_{j l m} \psi_{l} h_{m} \\
& +\sum_{l} b_{j l} \psi_{l}-\mu \lambda_{j}\left(\psi_{j}-\psi_{j}^{*}\right),
\end{aligned}
$$

where

$$
\begin{aligned}
c_{j l m} & \equiv \frac{1}{2 \pi^{2} b} \int \bar{\Phi}_{j} J\left(\Phi_{l}, \Phi_{m}\right) d x d y \\
b_{j l} & \equiv \frac{1}{2 \pi^{2} b} \int \bar{\Phi}_{j} J\left(\Phi_{l}, f\right) d x d y,
\end{aligned}
$$

with $J$ denoting the advection Jacobian defined by (9) and overbars denoting complex conjugates. Expressions for the coefficients $c_{i j k}$ and $b_{j l}$ are given in the appendix.

Assuming simple forms for the topography and forcing, truncating (13) to $\left|j_{1}\right|, j_{2} \leq 2$, and substituting an expansion in a real basis $\Psi(x, y, t)=\Sigma_{i} \mathrm{x}_{i}(t) Y_{i}(x$, $y$ ) for the expansion (13), where the new basis functions are given in Table 5, de Swart (1988, 1989) obtained a 10-component dynamical system. A problem with the de Swart model is that it requires unrealistically large values of the thermal forcing $\Psi^{*}$, and hence of the background winds $u=-\Psi_{y}^{*}$, to avoid stable fixed points. This problem can be regarded as an artifact of the truncation and of the $\beta$ plane approximation since Legras and Ghil (1985), for instance, constructed a similar model with more modes and with spherical geometry that behaves appropriately for realistic values of the forcing. Therefore, for the purpose of studying relationships between the two hemispheres qualitatively, we adopt the simpler de Swart model.

A typical trajectory of the system (14) is represented in Fig. 9 by a time series of values of the amplitude $x_{1}$ of the gravest zonal flow mode. Regime structure is manifested as different ranges of oscillation in different portions of the time series. A careful analysis (de Swart 1988, 1989) actually reveals three regimes corresponding to three unstable fixed points, but two regimes are easily discerned in the figure. The value of the variable 


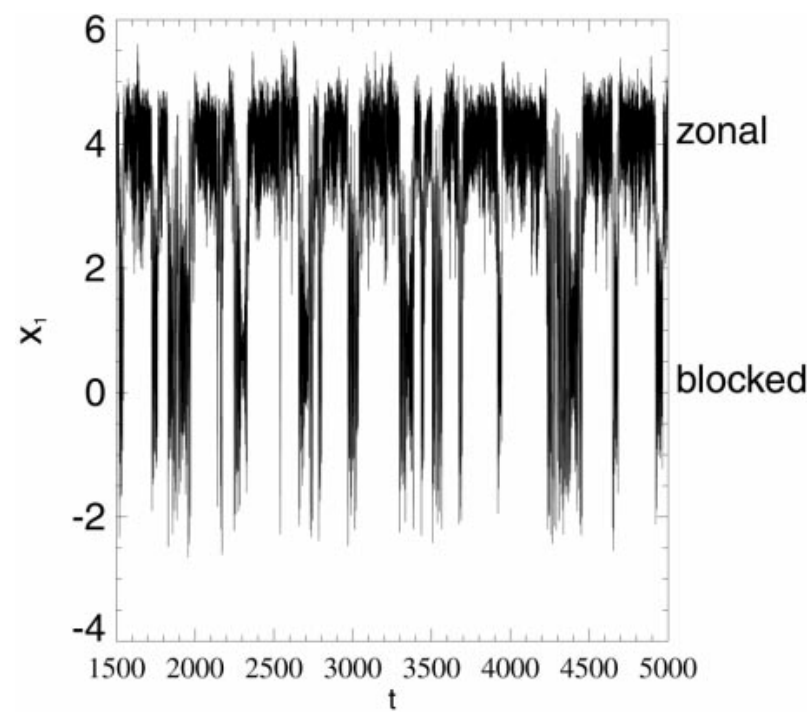

FIG. 9. A typical trajectory of the 10-component de Swart $(1988,1989)$ model of the midlatitude atmospheric circulation, specified by projecting ( 8 ) onto the eigenfunctions given in Table 5 . The values $\mathrm{x}_{1}$ are plotted vs $t$ for an adaptive Runge-Kutta numerical integration with nondimensional parameters: $\beta=1.25, \mu=0.1, \gamma$ $=1, b=1.6, \mathrm{x}_{1}^{*}=4$, and $\mathrm{x}_{4}^{*}=-8$, where the thermal forcing is given by $\Psi^{*}=\sum_{i=1,4} X_{i}^{*}(t) Y_{i}$. This corresponds to $f_{0}$ and $\beta$ at $45^{\circ}$ latitude, a topographic height of $1 \mathrm{~km}$, a dissipation timescale of 12 days, and background winds of up to $210 \mathrm{~m} \mathrm{~s}^{-1}$. Only $t>1500$ (in nondimensional time units) is displayed so as to exclude transients.

$\mathrm{X}_{1}$ is sufficient to distinguish between these regimes, which arguably correspond to zonal and blocked flow, respectively, though the regime residence times in the wavenumber 2 model are not realistic. Typical flow structures for the two regimes are shown in Fig. 10.

\section{b. The two-hemisphere model}

The two midlatitude systems are coupled, in the quasigeostrophic approximation, because of the exchange of Rossby waves through the Tropics. However, a meridionally propagating Rossby wave only penetrates the Tropics in longitudinal bands where the average zonal winds $\bar{u}$ are westerly (Webster and Holton 1982), as illustrated in Fig. 7 (for a numerical model more highly resolved than the models to be considered here). More generally, a propagating wave with zonal phase speed $c_{x} \neq 0$ is either reflected or absorbed when it reaches a critical line where $\bar{u}-c_{x}=0$. The critical line is thus defined by a value of $\bar{u}$, giving the requisite "Doppler shift," which is different for different wave modes. The existence of the critical line, ignoring the mode dependence, is the reason for the boundary condition (10a) on the tropical side of the channel. As illustrated in Fig. 8, the westerly ducts that break the tropical boundary are regions typically no more than $45^{\circ}$ in longitudinal extent that form intermittently over the Atlantic and Pacific Oceans during boreal winters. a)

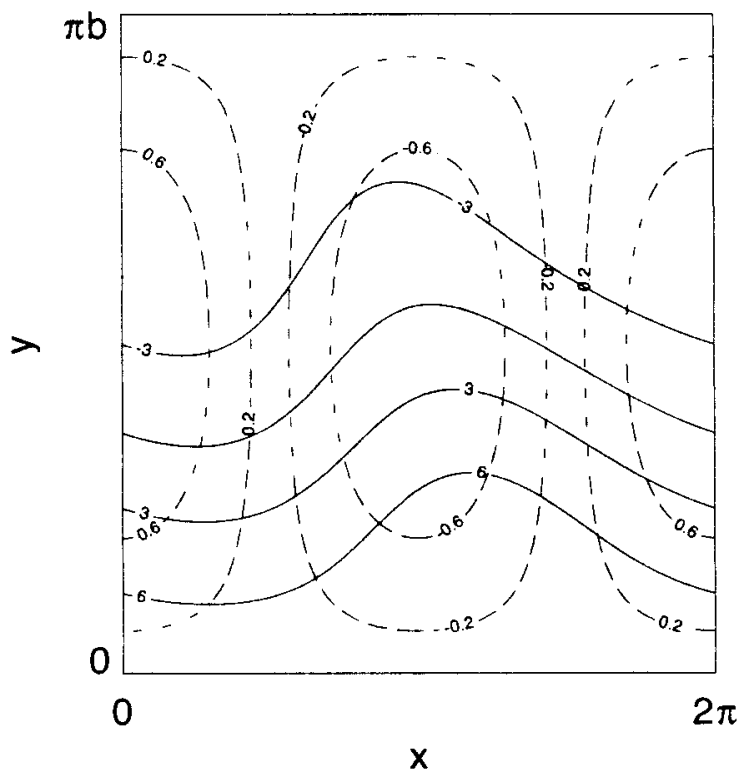

b)

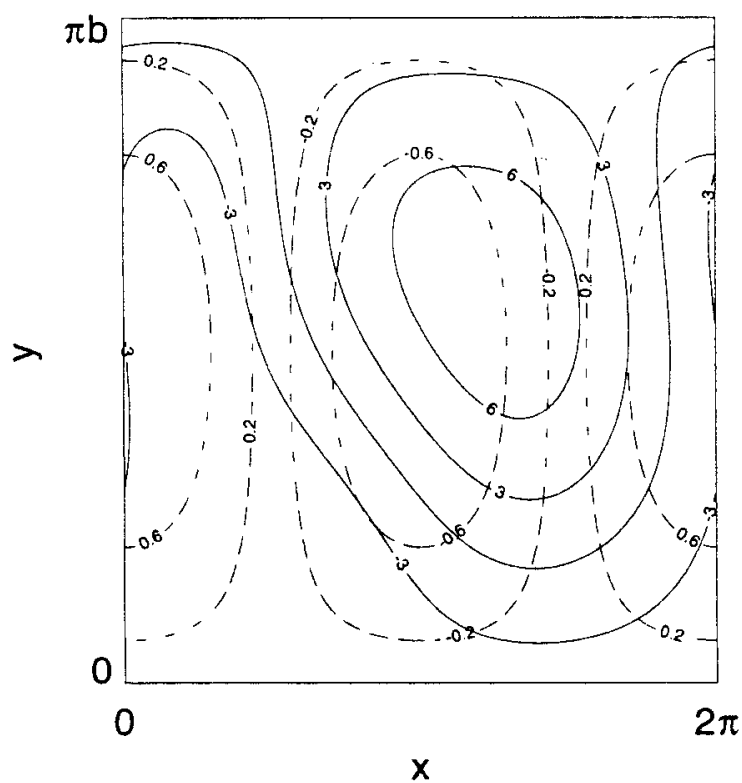

FIG. 10. Streamfunction in nondimensional units in an integration of the de Swart $(1988,1989)$ model at typical instants when the flow is (a) zonal, with $x_{1}=4.0$, and (b) blocked, with $x_{1}=-1.7$. (One nondimensional unit is $1.1 \times 10^{6} \mathrm{~m}^{2} \mathrm{~s}^{-1}$.) The topographic height in kilometers is also shown (dotted contours). 
To the extent that the boundary conditions in (10) accurately represent the physical effects of the tropical easterly barrier, the same boundary conditions with holes can be used to construct a model of the two midlatitude systems, coupled through the westerly ducts.

Duane (1997) formulated the coupling between the two midlatitude systems that is engendered by the transmission of Rossby waves through the westerly ducts, by neglecting the meridional extent of the region of tropical easterlies, taking this region to be a line at the equator, and also assuming that the two midlatitude systems lay on a single $\beta$ plane. The former assumption will be relaxed in section $3 \mathrm{c}$, where we consider transmission across an extended tropical region. The latter assumption, which corresponds to $f$ varying linearly with latitude everywhere, would be exact on a planet with a shape that is not spherical but that is also not terribly unrealistic.

The effect of the opposite hemisphere is that of a boundary forcing in the longitudinal range corresponding to the tropical westerly ducts. The forcing is given by replacing the boundary condition of zero meridional wind by the condition that meridional winds match across the tropical boundary, that is, that $v\left(x, 0^{+}\right)=$ $v\left(x, 0^{-}\right)$in the duct regions or equivalently that $\partial \Psi / \partial x$ matches across the boundary in these regions.

To isolate the effects of the boundary forcing in a linear equation that can be solved by conventional methods, the streamfunction $\Psi$ is expressed as the sum of two parts: $\Psi=\Psi_{0}+\Psi_{B}$. The boundary-forced, or "diffracted," part $\Psi_{B}$ is a solution of the linearized vorticity equation:

$$
\frac{\partial\left(\nabla^{2} \Psi_{B}\right)}{\partial t}+J\left(\Psi_{B}, f\right)+\mu \nabla^{2} \Psi_{B}=0,
$$

with matching boundary conditions, as described above, in the duct regions. The remaining free part $\Psi_{0}$ satisfies the Charney-Devore boundary conditions in (10) and is such that the total streamfunction $\Psi$ satisfies the full vorticity equation (8). The diffracted streamfunction $\Psi_{B}$ is posited to satisfy a specific inhomogeneous condition on the tropical boundary: in the duct regions, $\Psi_{B}$ is set equal to the sum of the southward-propagating part of the total flow north of the boundary and the northwardpropagating part of the total flow south of the boundary. That is,

$$
\Psi_{B}^{\mathrm{N}}(x, 0, t)=\Psi_{B}^{\mathrm{s}}(x, 0, t)=\left\{\begin{array}{ll}
0 & \text { outside ducts } \\
T(t) \sum_{j} \hat{\psi}_{j}^{\mathrm{N}}(t) \Phi_{j}^{\downarrow}(x, 0)+\hat{\psi}_{j}^{\mathrm{s}}(t) \Phi_{j}^{\uparrow}(x, 0) & @ \text { ducts }
\end{array} \equiv D(x, t),\right.
$$

where $T$ is a parametric transmission coefficient, perhaps time dependent, and the coefficients $\hat{\psi}_{j}^{\mathrm{N}}$ and $\hat{\psi}_{j}^{\mathrm{S}}$ are the coefficients in the Charney-Devore/de Swart spectral truncation of the full streamfunction in either hemisphere: $\Psi^{\mathrm{N}, \mathrm{S}}=\Sigma_{j} \psi_{j}^{\mathrm{N}, \mathrm{S}} \Phi_{j}^{\mathrm{N}, \mathrm{S}}$. The modes $\Phi_{j}^{\mathrm{N}, \mathrm{S}}$ are chosen to have symmetry properties such that the single-hemisphere equations derived from (14) will be the same on both sides of the equator. The northward- and southward-propagating parts of the Rossby modes $\Phi_{j}=$ $\sqrt{2} e^{i j_{1} x} \sin \left(j_{2} y / b\right),\left|j_{1}\right|, j_{2}>0$ are given by

$$
\begin{aligned}
\Phi_{j} & =\Phi_{j}^{\uparrow}+\Phi_{j}^{\downarrow} \\
\Phi_{j}^{\uparrow} & = \pm \frac{i}{\sqrt{2}} \exp \left(i j_{1} x \mp i j_{2} \frac{y}{b}\right) \\
\Phi_{j}^{\downarrow} & =\mp \frac{i}{\sqrt{2}} \exp \left(i j_{1} x \pm i j_{2} \frac{y}{b}\right),
\end{aligned}
$$

where the upper (lower) signs apply when $j_{1}>0, \omega_{j}<$ $0\left(j_{1}<0, \omega_{j}>0\right)$. The zonal flow modes $\Phi_{0_{j} j_{2}}=\sqrt{2}$ $\cos \left(j_{2} y / b\right)$, with $\omega_{j}=0$, have no northward- or southward-propagating parts. Details of the remaining boundary conditions and north-south symmetries can be found in Duane (1997). Because of our assumptions regarding the shapes of the ducts and the background wind field, however, the sizes of the ducts and the transmission coefficient $T$ are not to be taken literally and are instead intended to parametrize the various effects, many of which we have neglected, that determine the strength and form of the physical coupling. The representation (16) of the physical coupling is illustrated schematically in Fig. 11. The various streamfunctions and spectral expansion coefficients that have been used in the foregoing or will enter the derivation are listed in Table 6 .

Since $\Psi_{B}$ satisfies the linear equation (15), it can be expressed as an integral, over the boundary and over history, of the boundary values multiplied by a boundary Green's function $G^{b}$ :

$\Psi_{B}(\mathbf{r}, t)=\int_{t_{0}<t} D\left(x_{0}, t_{0}\right) G^{b}\left(\mathbf{r}, t \mid x_{0}, t_{0}\right) d x_{0} d t_{0}$,

where $\mathbf{r}=(x, y), \mathbf{r}_{0}=\left(x_{0}, y_{0}\right)$, and we have introduced the single streamfunction $\Psi_{B}(x, y, t) \equiv \Psi_{B}^{\mathrm{N}}(x, y, t)=$ $\Psi_{B}^{\mathrm{S}}(x,-y, t)$. A spectral form for the boundary Green's function is derived in Duane (1997): 


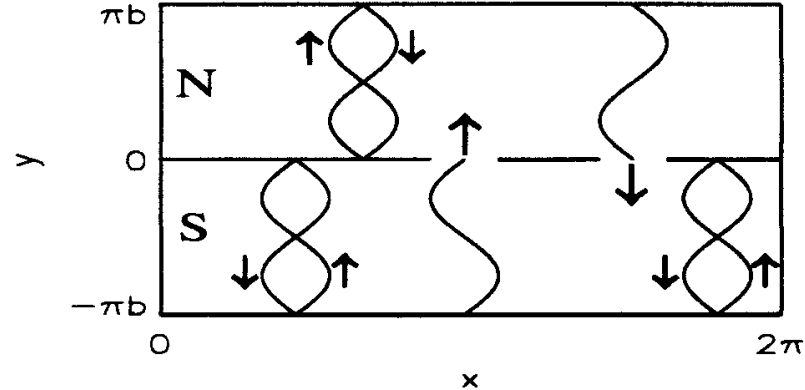

FIG. 11. Schematic diagram of the two-hemisphere model. The tropical easterly barrier is assumed infinitely thin, and both hemispheres are taken to lie on a single $\beta$ plane. The two midlatitude systems are boundary-forced by the outward-propagating components of the meridionally standing wave modes in each hemisphere.

$$
\begin{aligned}
& G^{b}\left(\mathbf{r}, t \mid x_{0}, t_{0}\right) \\
& =-i \theta\left(t-t_{0}\right) \frac{1}{2 \pi^{2} b} \sum_{j_{1} \neq 0} \frac{\omega_{j} \frac{\partial \Phi_{j}}{\partial y}\left(x_{0}, 0, t_{0}\right) \Phi_{j}(\mathbf{r})}{\lambda_{j}} \\
& \quad \times \exp \left[\left(-i \omega_{j}-\mu\right)\left(t-t_{0}\right)\right],
\end{aligned}
$$

where, as before, $\omega_{j}=-j_{1} \beta / \lambda_{j}, \lambda_{j}=j_{1}^{2}+j_{2}^{2} / b$ are the eigenvalues of $\nabla^{2} \Phi_{j}=-\lambda_{j} \Phi_{j}$, and $\theta$ is the unit step function.

Note that if we substitute (19) in the integral in (18) we get a sum of eigenmodes for homogeneous boundary conditions (multiplied by overlap integrals) as a purported solution to the linearized equation with inhomogeneous boundary conditions. Our method (Morse and Feshbach 1953) will give errors in a boundary layer near the ducts, which will narrow as we retain an increasing number of terms in the expansion.

All streamfunctions are expanded in eigenmodes:

$$
\begin{aligned}
\Psi_{B} & =\sum_{j_{1} \neq 0} \zeta_{j}(t) \Phi_{j} \\
\Psi^{\mathrm{N}, \mathrm{S}} & =\sum_{j} \hat{\psi}_{j}^{\mathrm{N}, \mathrm{S}} \Phi_{j}^{\mathrm{N}, \mathrm{S}} \\
\Psi_{0}^{\mathrm{N}, \mathrm{S}} & =\sum_{j} \psi_{j}^{\mathrm{N}, \mathrm{S}} \Phi_{j}^{\mathrm{N}, \mathrm{S}} .
\end{aligned}
$$

The sum in (20) is also seen to be over Rossby modes only by substitution of (19) in (18). The structure of the two-hemisphere model, schematized in Fig. 12, resembles the structure of low-order systems that have previously been reported to exhibit synchronized chaos (Pecora and Carroll 1990; Duane 1997) in that some but not all of the dynamical variables are coupled. The physical reason for the decoupling of the zonal flow modes defined in (11a) is that the zonal flow vanishes at the interface, that is, $u=-\partial \Phi_{j} / \partial y=0$ for these modes at $y=0$, so they do not contribute to the spectral expansion of $G^{b}$ in (19). Since the Rossby modes are odd in $y, \Psi_{B}(x,-y, t)=-\Psi_{B}(x, y, t)$, so the decom-
TABLE 6. Streamfunctions and spectral expansion coefficients used in the derivation of the low-order models. Coefficients are for expansions in the complex basis functions $\Phi_{j}$ except where indicated. Quantities for the two-hemisphere model are listed without

\begin{tabular}{|c|c|c|}
\hline \multicolumn{2}{|c|}{ Streamfunction coefficients } & \\
\hline \multicolumn{3}{|c|}{ Basis functions } \\
\hline$\Phi_{j}(x, y)$ & & $\begin{array}{l}\text { complex eigenfunction of } \nabla^{2} \text { with } \\
\text { eigenvalue }-\lambda\end{array}$ \\
\hline $\bar{\Phi}_{j}(x, y)$ & & $\begin{array}{l}\text { complex conjugate of eigenfunc- } \\
\text { tion } \Phi_{j}\end{array}$ \\
\hline$\Upsilon_{i}(x, y)$ & & $\begin{array}{l}\text { real eigenfunction of } \nabla^{2} \text { defined in } \\
\quad \text { Table } 5 \text { for } i=1, \ldots, 10\end{array}$ \\
\hline \multicolumn{3}{|c|}{ Single-hemisphere model } \\
\hline$\Psi(x, y, t)$ & $\left\{\begin{array}{c}\psi_{j}(t)(\mathrm{com}- \\
\text { plex basis }) \\
x_{i}(t) \\
\text { (real basis) }\end{array}\right.$ & $\left\{\begin{array}{l}\text { solution of barotropic vorticity } \\
\text { equation with homogeneous } \\
\text { boundary conditions }\end{array}\right.$ \\
\hline \multicolumn{3}{|c|}{ Coupled-hemisphere model } \\
\hline$\Psi_{B}(x, y, t)$ & $\zeta_{j}(t)$ & $\begin{array}{l}\text { solution of linearized barotropic } \\
\text { vorticity equation with boundary } \\
\text { forcing } \psi_{B}(x, 0, t)=D(x, t)\end{array}$ \\
\hline$\Psi(x, y, t)$ & $\hat{\psi}_{j}(t)$ & $\begin{array}{l}\text { solution of barotropic vorticity } \\
\text { equation with boundary forcing }\end{array}$ \\
\hline$\Psi_{0}(x, y, t)$ & $\psi_{j}(t)$ & $\begin{array}{l}\psi-\psi_{B} \text { (satisfies homogeneous } \\
\text { boundary conditions) }\end{array}$ \\
\hline$\Phi_{j}^{\uparrow}(x, y)$ & & $\begin{array}{l}\text { northward-propagating part of ei- } \\
\text { genmode } \Phi_{j}\end{array}$ \\
\hline$\Phi_{j}^{\downarrow}(x, y)$ & & $\begin{array}{l}\text { southward-propagating part of ei- } \\
\text { genmode } \Phi_{j}\end{array}$ \\
\hline$\Psi *(y, t)$ & $\psi_{j}^{*}(t)$ & $\begin{array}{l}\text { zonal flow driven by equator-to- } \\
\text { pole temperature gradient (in } \\
\text { model with annual cycle, the co- } \\
\text { efficients vary sinusoidally with } \\
\text { average values } \bar{\psi}_{j} \text { and variation } \\
\text { amplitude } d_{j} \text { ) }\end{array}$ \\
\hline
\end{tabular}
the superscripts $\mathrm{N}$ and $\mathrm{S}$ used in the text, which indicate the corresponding quantities in the Northern and Southern Hemispheres, respectively. The subscript $j$ is shorthand for the pair of indices $\left(j_{1}\right.$, $j_{2}$ ) throughout.

position of the total streamfunction in the two hemispheres becomes

$$
\begin{aligned}
& \Psi^{\mathrm{N}}(x, y, t)=\Psi_{0}^{\mathrm{N}}(x, y, t)+\Psi_{B}(x, y, t) \\
& \Psi^{\mathrm{s}}(x, y, t)=\Psi_{0}^{\mathrm{S}}(x, y, t)-\Psi_{B}(x, y, t),
\end{aligned}
$$

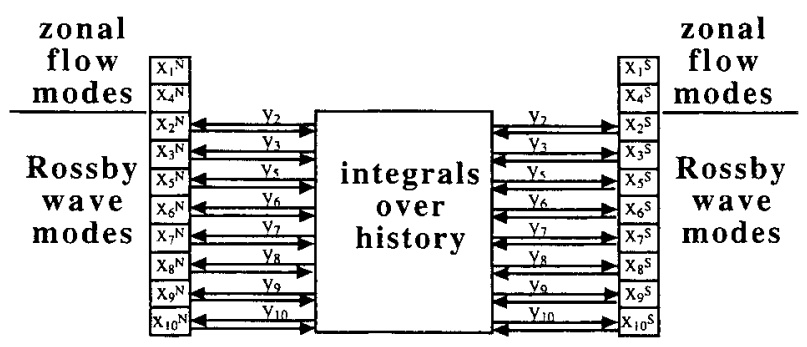

FIG. 12. Schematic representation of the structure of the truncated 28-component model of the coupled hemispheres. Zonal flow modes are uncoupled, while each Rossby mode is coupled to all Rossby modes in the opposite hemisphere at all past times. 
or, in terms of the time-dependent expansion coefficients,

$$
\begin{aligned}
& \hat{\psi}_{j}^{\mathrm{N}}=\psi_{j}^{\mathrm{N}}+\zeta_{j} \\
& \hat{\psi}_{j}^{\mathrm{S}}=\psi_{j}^{\mathrm{S}}-\zeta_{j} .
\end{aligned}
$$

The boundary forcing in (16) is seen to be $D(x)=$ $T \Sigma_{k_{1}, k_{2}} \Phi_{k}^{\uparrow}(x, 0)\left(\hat{\psi}_{k}^{\mathrm{N}}-\hat{\psi}_{k}^{S}\right)$ at the longitudes of the ducts (with time dependence suppressed), where we have noted from (17) that $\Phi_{k}^{\uparrow}(x, 0)=-\Phi_{k}^{\downarrow}(x, 0)$. The coefficients of the modes in $\Psi_{B}$ are then found from (18) and (19):

$$
\zeta_{j}(t)=\int_{-\infty}^{t} e^{\left(-i \omega_{j}-\mu\right)\left(t-t_{0}\right)} \frac{1}{2 \pi^{2} b} \sum_{k_{1}, k_{2}} \int_{x \in \text { ducts }}\left(\frac{\partial}{\partial y} \bar{\Phi}_{j}(x, 0)\right) \Phi_{k}^{\uparrow}(x, 0) \frac{-i \omega_{j} T\left(\hat{\psi}_{k}^{\mathrm{N}}\left(t_{0}\right)-\hat{\psi}_{k}^{\mathrm{S}}\left(t_{0}\right)\right)}{\lambda_{j}} d x d t_{0}
$$

or, differentiating,

$$
\dot{\zeta}_{j}=\left(-i \omega_{j}-\mu\right) \zeta_{j}-i \frac{\omega_{i}}{\lambda_{j}} \frac{T}{2 \pi^{2} b} \sum_{k_{1}, k_{2}} W_{j k}\left(\hat{\psi}_{k}^{\mathrm{N}}-\hat{\psi}_{k}^{\mathrm{S}}\right)
$$

where we have introduced notation for the overlap integrals:

$$
W_{j k} \equiv \int_{x \in \text { ducts }}\left(\frac{\partial}{\partial y} \bar{\Phi}_{j}(x, 0)\right) \Phi_{k}^{\uparrow}(x, 0) d x .
$$

By subtracting (15) from (8), it is seen that firstorder differential equations for the coefficients of the modes in the two-hemisphere model can be obtained by substituting $\psi_{j}$ for $\psi_{j}$ in all terms of the singlehemisphere equations (14) except the time-derivative, $\beta$-effect, and dissipation $(\sim \mu)$ terms, and then including (25). Thus the equations of the two-hemisphere model are

$$
\begin{aligned}
\lambda_{j} \dot{\psi}_{j}^{\mathrm{N}}= & \frac{1}{2} \sum_{l, m \in S_{K}} c_{j l m}\left(\lambda_{l}-\lambda_{m}\right) \hat{\psi_{l}^{\mathrm{N}}} \hat{\psi}_{m}^{\mathrm{N}}+\gamma \sum_{l, m \in S_{K}} c_{j l m} \hat{\psi}_{l}^{\mathrm{N}} h_{m}^{\mathrm{N}} \\
& +\sum_{l \in S_{K}} b_{j l} \psi_{l}^{\mathrm{N}}-\mu \lambda_{j}\left(\psi_{j}^{\mathrm{N}}-\psi_{j}^{\mathrm{N} *}\right) \\
\lambda_{j} \dot{\psi}_{j}^{\mathrm{S}}= & \frac{1}{2} \sum_{l, m \in S_{K}} c_{j l m}\left(\lambda_{l}-\lambda_{m}\right) \hat{\psi_{l}^{\mathrm{S}}} \hat{\psi}_{m}^{\mathrm{s}}+\gamma \sum_{l, m \in S_{K}} c_{j l m} \hat{\psi}_{l}^{\mathrm{s}} h_{m}^{\mathrm{S}} \\
& +\sum_{l \in S_{K}} b_{j l} \psi_{l}^{\mathrm{S}}-\mu \lambda_{j}\left(\psi_{j}^{\mathrm{s}}-\psi_{j}^{\mathrm{S} *}\right) \\
\dot{\zeta}_{j}= & \left(-i \omega_{j}-\mu\right) \zeta_{j}-i \frac{\omega_{j}}{\lambda_{j}} \frac{T}{2 \pi^{2} b} \sum_{k \in S_{K}} W_{j k}\left(\hat{\psi}_{k}^{\mathrm{N}}-\hat{\psi}_{k}^{\mathrm{S}}\right),
\end{aligned}
$$

where we have also truncated to wavenumber $K$ in both the meridional and zonal dimensions by restricting the summations to the sets $S_{K} \equiv\left\{\left(j_{1}, j_{2}\right) \mid-K \leq j_{1} \leq K, 1\right.$ $\left.\leq j_{2} \leq K\right\}$. For the case $K=2$, the system in (27a)(27c) was reduced to a set of 28 equations for real dynamical variables in Duane (1997).

The dynamical relationship between the boundaryforced streamfunction $\Psi_{B}$ and the total streamfunction $\Psi$ in the model (27a)-(27c) engenders dissipative coupling. That is, the magnitudes of the spectral coeffi- cients $\zeta_{j}$ for $\Psi_{B}$ approach quantities proportional to the differences between corresponding spectral coefficients $\hat{\psi}_{k}^{\mathrm{N}}$ and $\hat{\psi}_{k}^{\mathrm{S}}$ for the total streamfunction $\Psi$ in the two hemispheres, according to $(27 \mathrm{c})$. But according to (23), the contribution of the $\zeta_{j}$ to the $\hat{\psi}_{j}$ should then tend to equalize the total streamfunctions in the two hemispheres. An important approximation in the construction of the two-hemisphere model was to neglect even modes (in $y$ ) of the whole system, corresponding to half-integer values of $j_{2}$ with a phase shift, which would satisfy the boundary conditions in (10) only at $y=\pi b$ and $y=-\pi b$. Such half-integer modes would have to be considered if the westerly ducts spanned the entire equator, for instance. These modes can be ignored in our model, however, if we assume that the forcing terms $\Psi^{\mathrm{N} *}$ and $\Psi^{\mathrm{s} *}$ in (27a) and (27b), which give the background zonal winds in the two hemispheres due to thermal forcing, do not include such modes. Then it can be verified that no wave-triad interactions [given by the terms in (27a) and (27b) that are bilinear in $\psi$ ] will generate them since integer values of $j_{2}$ yield only integer sums and differences. It appears to us that the assumed form of the forcing (11), (13), which preserves the structure of the single-hemisphere models, is sufficiently general for the synchronization study since forcing terms possessing any symmetry across the equator, odd or even, can still be represented with the modes we have used.

\section{c. Coupling of the hemispheres across an extended tropical region}

If we regard the single-hemisphere model formulated in section $4 \mathrm{a}$ as describing the midlatitude region proper, so that the tropical easterly barrier has a finite latitudinal extent, then the Rossby waves linking the two hemispheres will be attenuated and phase-shifted as they propagate through the westerly ducts. The phase shift of a wave mode of the form (11b) through a duct of path length $\mathcal{P}$ is $\mathcal{P j}_{2} / b$. The attenuation of the mode over the same distance, arising from the friction term $\mu \nabla^{2} \Psi$ in the barotropic vorticity equation (8), is $\exp \left(-\mathcal{P} \mu \lambda_{j} / c_{y j}\right)$, where $c_{y}$ is the meridional phase speed 
of the wave. From (11b) and (12), this phase speed is $c_{y} \equiv \omega / k_{y}=\left(\beta j_{1} / \lambda_{j}\right) /\left(j_{2} / b\right)$. The phase shift and attenuation of the Rossby modes affect the boundary forcing of each midlatitude system by the opposite hemisphere, but the forcing due to the outgoing waves is unchanged.
Furthermore, the forcing due to incoming waves at time $t$ is determined by the values of the wave modes on the boundary of the opposite midlatitude region (before phase shifting and attenuation) at time $t-\mathscr{P} / c_{y}$. Therefore, (16) is replaced by

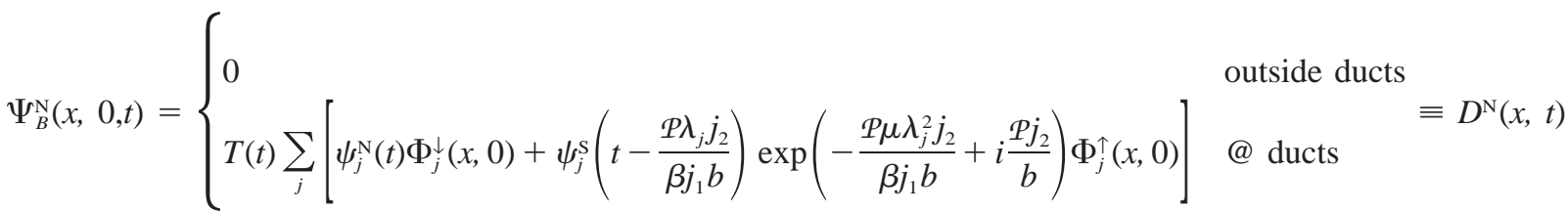

$$
\begin{aligned}
& \Psi_{B}^{\mathrm{S}}(x, 0, t)= \begin{cases}0 & \text { outside ducts } \\
T(t) \sum_{j}\left[\psi_{j}^{\mathrm{S}}(t) \Phi_{j}^{\uparrow}(x, 0)+\psi_{j}^{\mathrm{N}}\left(t-\frac{\mathcal{P} \lambda_{j} j_{2}}{\beta j_{1} b}\right) \exp \left(-\frac{\mathcal{P} \mu \lambda_{j}^{2} j_{2}}{\beta j_{1} b}-i \frac{\mathcal{P j _ { 2 }}}{b}\right) \Phi_{j}^{\downarrow}(x, 0)\right] & @ D^{\mathrm{S}}(x, t) .\end{cases}
\end{aligned}
$$

While the boundary forcing in (16) engenders transmission and reflection of waves at the boundary, the form (28) models absorption as well.

Since the two hemispheres are no longer forced by the same quantity $D(x, t)$ as in (16), we no longer have $\Psi_{B}^{\mathrm{N}}(x, y, t)=\Psi_{B}^{\mathrm{S}}(x,-y, t)$, so $\Psi_{B}^{\mathrm{N}}$ and $\Psi_{B}^{\mathrm{S}}$ must be regarded as separate dynamical variables. It follows that the low-order truncated models in the case of an extended tropical region will also have higher dimension than their counterparts in the previous section. Specifically, we must introduce distinct variables $\zeta_{j}^{\mathrm{N}}$ and $\zeta_{j}^{s}$ for the two hemispheres, respectively, by analogy with (20). The reasoning that led to (27) now leads to the following system of ordinary differential equations with delays:

$$
\begin{aligned}
\lambda_{j} \dot{\psi}_{j}^{\mathrm{N}}= & \frac{1}{2} \sum_{l, m \in S_{K}} c_{j l m}\left(\lambda_{l}-\lambda_{m}\right) \hat{\psi_{l}^{\mathrm{N}}} \hat{\psi}_{m}^{\mathrm{N}}+\gamma \sum_{l, m \in S_{K}} c_{j l m} \hat{\psi}_{l}^{\mathrm{N}} h_{m}^{\mathrm{N}} \\
& +\sum_{l \in S_{K}} b_{j l} \psi_{l}^{\mathrm{N}}-\mu \lambda_{j}\left(\psi_{j}^{\mathrm{N}}-\psi_{j}^{\mathrm{N} *}\right) \\
\lambda_{j} \dot{\psi}_{j}^{\mathrm{S}}= & \frac{1}{2} \sum_{l, m \in S_{K}} c_{j l m}\left(\lambda_{l}-\lambda_{m}\right) \hat{\psi}_{l}^{\mathrm{S}} \hat{\psi}_{m}^{\mathrm{S}}+\gamma \sum_{l, m \in S_{K}} c_{j l m} \hat{\psi}_{l}^{\mathrm{S}} h_{m}^{\mathrm{s}} \\
& +\sum_{l \in S_{K}} b_{j l} \psi_{l}^{\mathrm{S}}-\mu \lambda_{j}\left(\psi_{j}^{\mathrm{s}}-\psi_{j}^{\mathrm{S} *}\right)
\end{aligned}
$$

where time arguments have been suppressed, except when involving delays, and

$$
\begin{aligned}
& \hat{\psi}_{j}^{\mathrm{N}}=\psi_{j}^{\mathrm{N}}+\zeta_{j}^{\mathrm{N}} \\
& \hat{\psi}_{j}^{\mathrm{S}}=\psi_{j}^{\mathrm{S}}+\zeta_{j}^{\mathrm{S}} .
\end{aligned}
$$

For a truncation to $K=2$, the system (29) consists of 36 real dynamical variables.

A difference between the two-hemisphere system with no explicit Tropics, (27), and the system with an extended Tropics is that the former system of equations is satisfied by solutions $\psi_{j}$ of the single-hemisphere system (14). That is, if $\psi_{j}$ solves (14), then $\psi_{j}^{\mathrm{N}}=\psi_{j}^{\mathrm{S}}=\psi_{j}$, $\zeta_{j}=0$ solves (27), for symmetrical topography $h$ and symmetrical thermal forcing $\Psi^{*}$. The equations for the two-hemisphere system with an extended Tropics, (29a)-(29d), on the other hand, are not satisfied by the single-hemisphere solutions $\psi_{j}$. Nevertheless, (29a)- 
TABLE 7. Joint probability matrices as in Table 1 but for an integration of the two-hemisphere model (27a)-(27c) truncated to $K=$ 2 over a period of $5 \times 10^{4}$ nondimensional time units, with transmission coefficient $T=1$ and other parameters as in Fig. 13, where the regimes are zonal flow and blocked flow, defined by $x_{1}>3$ and $\mathrm{x}_{1}<3$, respectively, in either hemisphere. Error bars at the $2 \sigma$ level were computed from five Monte Carlo simulations. The $p$ value was computed by assuming a decorrelation time of 60 nondimensional time units.

\begin{tabular}{|c|c|c|c|c|}
\hline \multirow[t]{3}{*}{ (a) } & \multicolumn{4}{|c|}{ (b) } \\
\hline & \multicolumn{2}{|r|}{$\mathbf{P}$} & \multicolumn{2}{|c|}{ Pind } \\
\hline & $\mathrm{N}$ zonal & $\mathrm{N}$ blocked & $\mathrm{N}$ zonal & $\mathrm{N}$ blocked \\
\hline S zonal & 0.59 & 0.15 & 0.56 & 0.17 \\
\hline $\mathrm{S}$ blocked & 0.18 & 0.08 & 0.20 & 0.06 \\
\hline & & $C=0.12 \pm 0.03$ & & $p=0.001$ \\
\hline
\end{tabular}

(29d) possess symmetrical solutions $\psi_{j}^{\mathrm{N}}=\psi_{j}^{\mathrm{S}}$. It can be verified that these symmetrical solutions are just the solutions of the single-hemisphere system, modified to include some absorption by the tropical boundary at the longitudes of the ducts and where the reflecting boundary at the Tropics is moved to a receded position at these longitudes. Coupling two such single-hemisphere models, so modified, is another way to derive the system (29a)-(29d).

\section{Interhemispheric correlations in the model}

Regime correlations in the model can be described in the same manner as they were for observed data in section 3. We now base our definition of blocking on the apparent regime structure of the trajectory in Fig. 9. We call subsystem $\mathrm{N}(\mathrm{S})$ zonal at any time $t$ if and only if $x_{1}^{N}(t)>3\left(x_{1}^{S}(t)>3\right)$. Otherwise we call subsystem N (S) blocked at time $t$. The separate identity of the seldom occupied "transitional" regime described in deSwart $(1988,1989)$ is ignored here. The joint probabilities $P_{i, j}$ for a simulation of the model, and the corresponding probability values $P_{i, j}^{\text {ind }}$ in the case where the two subsystems individually have the same statistics but are assumed independent, defined as in (6), are given in Table 7. As with the observed data, the diagonal elements of the matrix $\mathbf{P}$ are larger than those of the matrix $P^{\text {ind }}$, while the off-diagonal elements are smaller, indicating correlation. We plot the standard measure of this correlation $\mathcal{C}$, which was defined in (7), as a function of the transmission coefficient $T$, assumed constant in time, in Fig. 13. The simulations used to compute $C$ were each conducted over a period equal to more than 800 decorrelation intervals, if we assume a maximal decorrelation time of about 60 nondimensional time units, corresponding to an average of regime-residence times reported by de Swart (1989). (This is much longer than the decorrelation time in the real atmosphere.) The correlations exceed the minimum correlation, shown in the figure, which would be significant at the $95 \%$ level according to chi-squared analysis. Typical error bars based on Monte Carlo estimates are also shown.

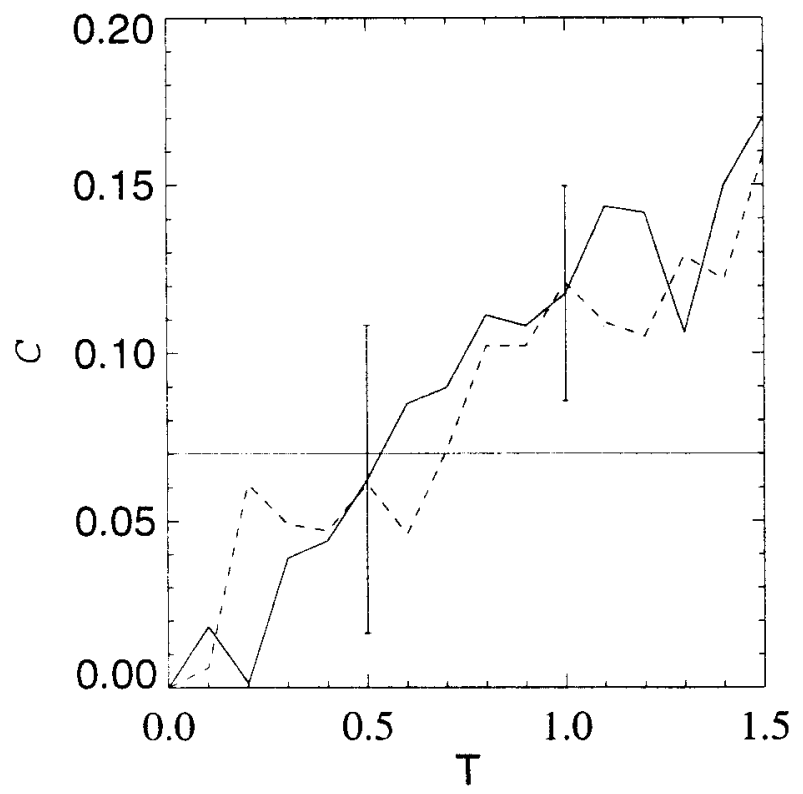

FIG. 13. The interhemispheric regime correlation $C$ as defined in (7), which quantifies the tendency for blocked states to co-occur, for varying interhemispheric transmission coefficient $T$, as defined in (16). Correlations are shown for the model (27a)-(27c) with symmetric topography, $h^{\mathrm{N}}=h^{\mathrm{S}}$ (solid line), and with more topography in the Northern Hemisphere, $h^{\mathrm{N}}=1.2, h^{\mathrm{S}}=0.85$ (dashed line). The value $C$ was computed from simulations over $5 \times 10^{4}$ nondimensional time units. The coefficients $W_{j k}$ in the equations (27a)-(27c) for the two-hemisphere system were chosen according to (26), for two ducts of width $\pi / 4$ (radians), separated by $\pi / 2$, then reducing the nondiagonal $(j \neq k)$ coefficients by half to allow for averaging over duct positions. Other parameters are as in Fig. 9, except that dimensional $f_{0}, \beta$ have their values at the equator and the dimensional values of the background winds range to $290 \mathrm{~m} \mathrm{~s}^{-1}$. Error bars at the 2- $\sigma$ level were computed from sets of five Monte Carlo simulations. The thin line is the minimum $C$ that would be significant at the $95 \%$ level according to chi-squared analysis, assuming a decorrelation time of 60 nondimensional time units.

While $T=1$ in Fig. 13 corresponds to a naive assumption about the nature of the ducts, as depicted in Fig. 11, and one might expect smaller values of $T$ in the realistic case that Rossby waves are attenuated, there is also evidence that realistically shaped ducts might serve to focus Rossby waves (Webster and Chang 1998), possibly giving an effective $T>1$. Additionally, there is the possibility of "tunneling" when the easterly barrier is narrow or when the easterly wind speeds are low, perhaps also giving an effective $T>1$. The coupling matrix $W_{j k}$ used in the simulations was first computed for two ducts of width $\pi / 4$ and separated by a distance of $\pi / 2$ in longitude. The factors $T$ and $W_{j k}$, however, should incorporate the effects of ducts of changing shape, size, position, and westerly wind speeds. Also, according to the Doppler-shift analysis in section $4 \mathrm{~b}$, "ducts" that transmit different modes have different boundaries. The values of the diagonal overlap integrals $W_{j j}$, given by (26), are less affected by such changes, for constant total duct width, than the nondiagonal elements $W_{j k}(j \neq k)$, which vanish when averaged over 


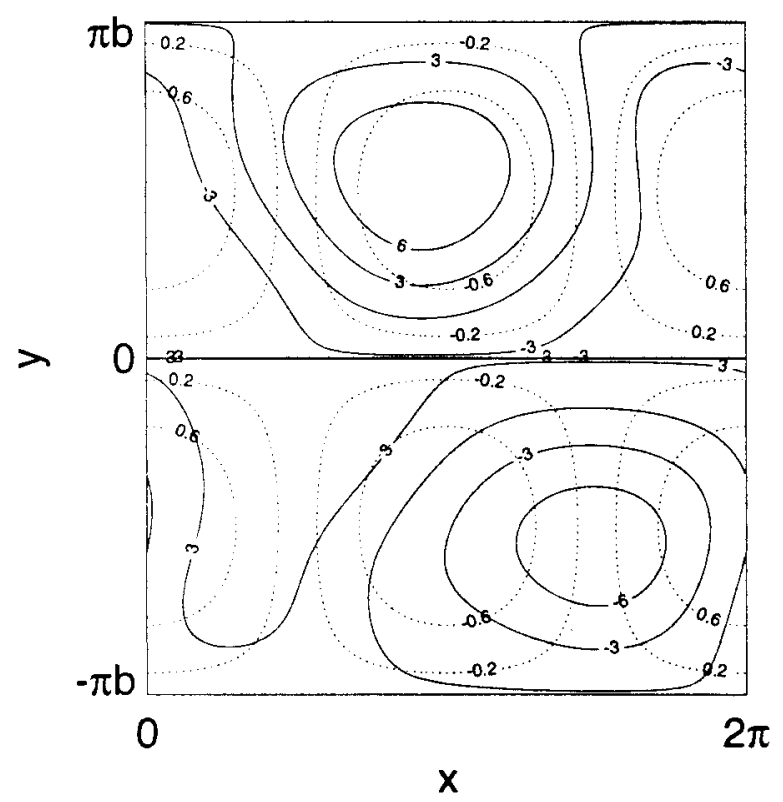

FIG. 14. Streamfunction and topographic height as in Fig. 10 but for the two-hemisphere model truncated to wavenumber 2, at an instant when both hemispheres are blocked. [A nondimensional streamfunction unit in the two-hemisphere model (27a)-(27c) is 2.2 $\times 10^{6} \mathrm{~m}^{2} \mathrm{~s}^{-1}$.] Parameters are as in Fig. 13, with $T=1$.

all possible duct configurations. Therefore, we employed the ad hoc device of reducing the values of the nondiagonal $W_{j k}$ by half in conducting the integrations. Individual hemisphere parameters are as given by de Swart (1988, 1989).

A typical flow pattern in which both hemispheres are blocked is shown in Fig. 14. Blocks tend to form at different longitudes because of the topographic configuration, which enters the dynamics with opposite signs of $f$ in the two hemispheres.

In Duane (1997), the correlations between blocking events in the two hemispheres were described as a manifestation of partially synchronized chaos. That is, it was shown in Duane (1997) that for very different, nonphysical values of the coupling parameters, the chaotic systems representing the two hemispheres would synchronize completely, that is, $\psi_{j}^{\mathrm{N}}(t)=\psi_{j}^{\mathrm{S}}(t)$, for all sufficiently large $t$, regardless of initial conditions. Apparently, the Rossby modes capture the "chaotic" behavior of each hemispheric system, so that coupling these modes is sufficient to drive the uncoupled zonal flow modes into synchrony. A bifurcation occurs as parameters begin to approach physical values, resulting in bursts of desynchronization that interrupt the synchronized phase at irregular intervals (Fig. 15), as is typical in other instances of synchronized chaos (Afraimovich et al. 1986; Venkataramani et al. 1996; Ashwin et al. 1994). Duane (1997) showed that the correlations detected at physical values of the parameters of $T$ and $W_{j k}$ can be regarded as a vestige of this synchronizing behavior and that such correlations are

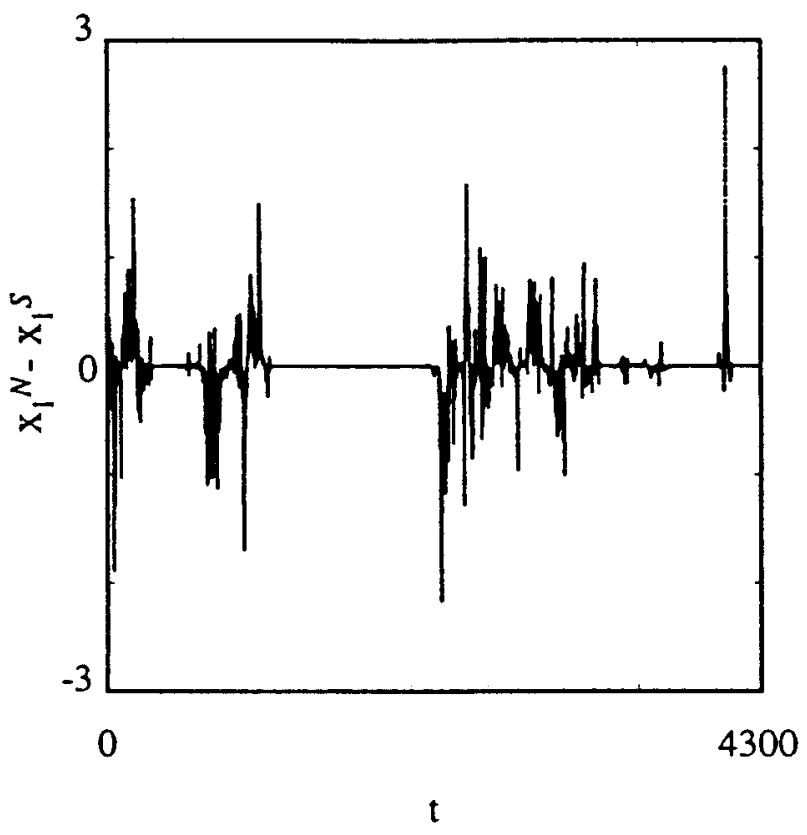

FIG. 15. Time series of differences between the trajectories of the two hemispheres in the coupled de Swart system [see (27a)-(27c)] with nonphysical parameters, represented by the difference $X_{1}^{N}-X_{1}^{S}$ between the coefficients of the gravest zonal flow modes in the two hemispheres, as plotted in Fig. 9. Parameters are as in Fig. 13 with $T=1$, except that the diagonal coefficients $W_{j k}, j=k$ are increased by a factor of 38 .

common in paired dynamical systems with mutually time-lagged coupling in a region of parameter space where the time lag associated with the coupling is the same as the intrinsic timescale of each component system. ${ }^{1}$ Here, we show that these correlations are maintained even if we abandon some of the unrealistic idealizations used in Duane (1997).

Figure 16 shows the regime correlation for the model with an extended tropical region for varying duct pathlength $\mathcal{P}$ in (29) expressed as a fraction of the latitudinal extent of each midlatitude region. It is seen that significant correlations persist for realistic duct lengths (compared, for instance, with the tropical barrier illustrated in Fig. 7.) This is expected, in view of the comments at the end of section $4 \mathrm{~d}$ regarding the reinterpretation of the extended Tropics model. The regime correlations can be regarded as arising from two Charney-Devore/de Swart type models coupled through a common boundary at the

\footnotetext{
${ }^{1}$ Kocarev et al. (1997) recently demonstrated synchronization in two one-dimensional systems, coupled at 32 evenly spaced junction points. This scheme implies that the time needed for a signal to propagate across an individual system is 32 times larger than the time needed for the coupling signal to reach any point of either system. Interestingly, this ratio of timescales is comparable to what Duane (1997) found was necessary to achieve synchronization in time-lagcoupled Lorenz systems and in the nonphysically coupled two-hemisphere model whose behavior is described in Fig. 15.
} 


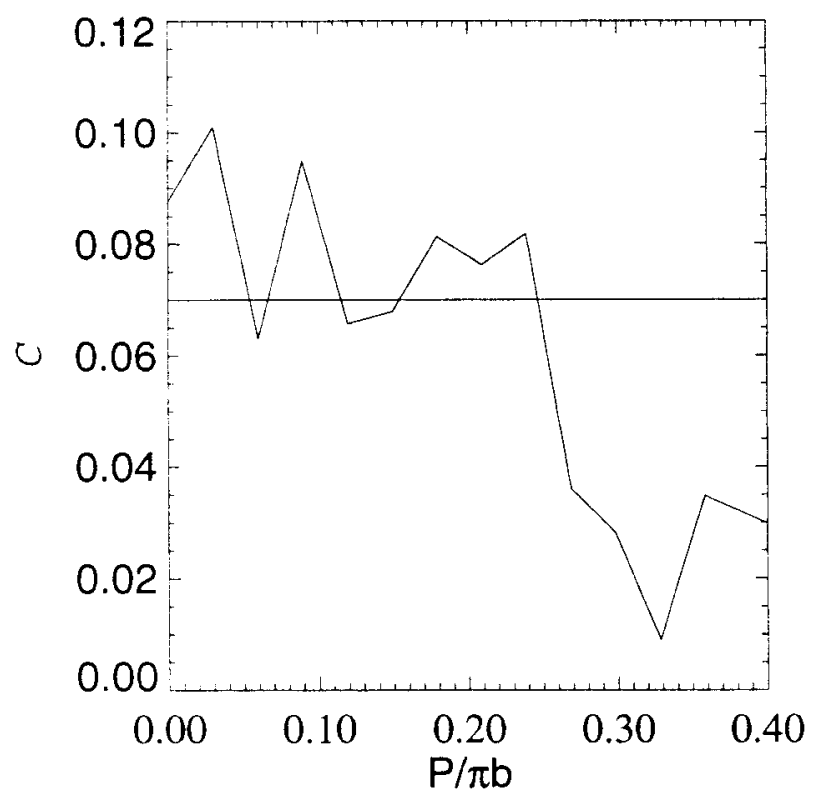

FIG. 16. The interhemispheric regime correlation $C$ as in Fig. 13 when Rossby wave signals linking the two midlatitude systems are phase-shifted and attenuated over a path length $\mathcal{P}$ in an extended tropical region, according to the modified two-hemisphere equations (29a)-(29d), with $T=0.75$. The path length on the horizontal axis is expressed as a fraction $\mathcal{P} / \pi b$ of midlatitude channel width. Other parameters are as in Fig. 13.

duct longitudes, as in the model with $P=0$, but with a tropical boundary for each midlatitude model that has a modified shape and reflection properties. If one believes that the original Charney-Devore model captured the qualitative features of the circulation in the midlatitudes, despite the idealizations in the boundary conditions, it is not surprising that the correlations observed in a coupled pair of such models should also be independent of these idealizations.

In formulating the generalized coupling (28) for the model with an extended Tropics, we chose to separate the effects of phase shift and attenuation from effects on either side of the equator associated with the specific shape of the ducts, which might ostensibly require Rossby waves to turn corners, for example. Ignoring such effects was consistent with our decision to ignore the detailed characteristics of the ducts, for example, due to varying background wind speeds in the $P=0$ model. In general, the effect of finite duct width on either side of the equator will be to reduce the parametric transmission coefficient $T$ by factors based on the geometry ( $T=0.75$ was used in Fig. 16), with consequences for the regime correlations that can be gleaned from Fig. 13.

Next, we show the dependence of the correlations on the relative positions of the topographic features in the two hemispheres. Figure 17 plots the regime correlation as a function of the longitudinal shift $\alpha$ in Southern Hemisphere topography, where the topography used to construct the two-hemisphere model (27) is

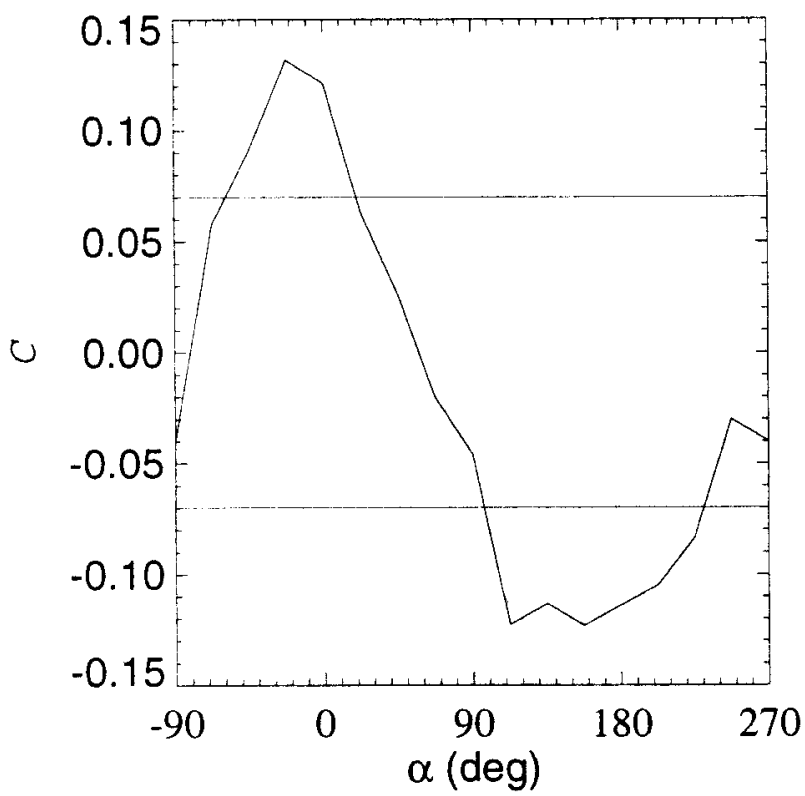

FIG. 17. The interhemispheric regime correlation $\mathcal{C}$ as in Fig. 13 when topographic features in the Southern Hemisphere are shifted longitudinally by $\alpha$, relative to the positions in Fig. 14. [The topography in each hemisphere is defined in (31).] The parameter $T=1$ and other parameters are as in Fig. 13. Here, the two thin lines represent the minimum significant correlation and the minimum significant anticorrelation.

$$
\begin{aligned}
& h^{\mathrm{N}}(x, y)=\cos (x) \sin \left(\frac{y}{b}\right) \\
& h^{\mathrm{S}}(x, y)=\cos (x+\alpha) \sin \left(\frac{y}{b}\right),
\end{aligned}
$$

which is then spectrally expanded according to (13). [The dimensional topography, as shown in Fig. 14, changes sign in the Southern Hemisphere so as to maintain the sign of the constant $\gamma$ multiplying the term for topographic $\beta$ effect in (8).] In Duane (1997), it was shown that the correlations were tolerant of some asymmetry in the magnitude of the topography, though this asymmetry caused the two hemispheres individually to behave differently. Significant correlations are shown in Fig. 13 (dashed line) for a model with enough topographic asymmetry as to roughly double blocking frequency in one hemisphere and halve it in the other. In the case of relative orientation of topographic features, on the other hand, the behavior of each hemisphere considered singly should be completely unchanged because of the zonal symmetry of the barotropic vorticity equation (8). Yet the correlation in Fig. 17 is seen to vary systematically with $\alpha$, becoming negative over as large a range of $\alpha$ as that for which it is positive.

A physical explanation of the anticorrelation between blocked states in the two hemispheres, for $\alpha=\pi$, is perhaps the following: in the de Swart model, the blocked flow regime is characterized by wavenumber 2 
blocks in a fixed position with respect to the "topographic" features (corresponding to continents in the original theory) in a given hemisphere. For $\alpha=0$, these block positions are the same as those implied by analytic continuation of the streamfunction in the opposite hemisphere, when that hemisphere is blocked. However, for $\alpha=\pi$, the synchronization tendency, when the opposite hemisphere is blocked, would cause blocks to form at positions opposite in longitude to those favored by the hemisphere's intrinsic dynamics. In this situation the compromise chosen is zonal flow in the given hemisphere, when the opposite hemisphere is blocked, hence anticorrelation.

One might want to determine an effective value of $\alpha$ for various theories of the forcing mechanism in the Southern Hemisphere, to see whether any of these theories are favored by the positive interhemispheric correlations actually observed. Coughlan (1983), for instance, noting the scarcity of topographic features in the Southern Hemisphere, has suggested that longitudinal temperature variation (ultimately due to the shape of the Antarctic continent) may play a role in forcing blocking behavior analogous to that of topography in the Northern Hemisphere. To compare theories of the forcing mechanism with our model, it would be necessary to reconcile the model structure with the observed statistics indicating two blocking centers in the Northern Hemisphere as compared to three in the Southern Hemisphere, probably due to corresponding differences in effective topographic wavenumber between the two hemispheres. Since the results for $h^{\mathrm{N}} \neq h^{\mathrm{s}}$ shown in Fig. 13 (dashed line) may be regarded as evidence that the correlations are robust against asymmetry in the magnitude of any given spectral component of the global "topography," it is suggested that the modeled correlations will also be consistent with different numbers of blocking centers.

The greatest asymmetry between the hemispheres (at a given instant of time) arises from the annual cycle, since the thermal forcing term $\Psi^{*}$ in the barotropic vorticity equation (8), essentially the geostrophic wind, varies with the equator-to-pole temperature difference. Additionally, the westerly ducts are only open during boreal winter, as shown in Fig.(8). We model the opening and closing of the westerly ducts with a time-dependent transmission coefficient:

$$
T(t)=\frac{1}{2} T_{0}(\sin (\nu t)+|\sin (\nu t)|),
$$

where $\nu=2 \pi /(1$ year $)=0.1991$ (in nondimensional units) and $T_{0}$ is an average value. The value $T$ as given by (32) vanishes during half the year and varies sinusoidally during the other half. The annual cycle in thermal forcing is modeled by introducing a time dependence in the forcing parameters $\psi^{\mathrm{N}, \mathrm{S} *}$ in (27):

$$
\begin{aligned}
\psi_{j}^{\mathrm{N} *} & =\bar{\psi}_{j}^{\mathrm{N} *}\left[1+d_{j} \sin (\nu t)\right] \\
\psi_{j}^{\mathrm{S}_{*}} & =\bar{\psi}_{j}^{\mathrm{S}_{*}}\left[1-d_{j} \sin (\nu t)\right],
\end{aligned}
$$

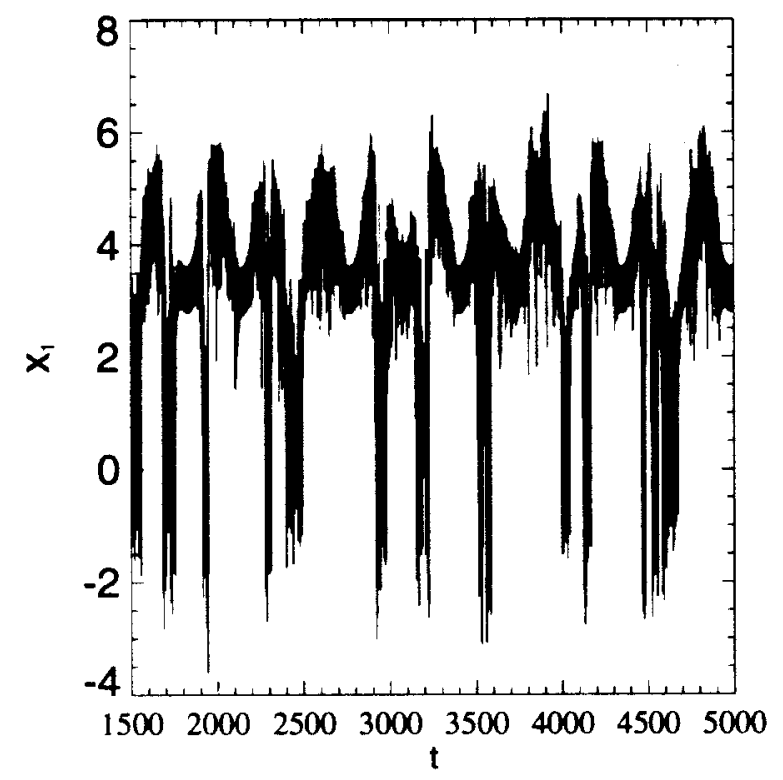

FIG. 18. Single-hemisphere trajectory as in Fig. 9 but with an annual cycle in thermal forcing given by (33a).

where the $d_{j}$ are constants that specify the asymmetry in each component $\psi_{j}^{*}$ of the forcing streamfunction. A typical single-hemisphere trajectory with an annual cycle (33) in thermal forcing is depicted in Fig. 18. With the values $d_{1}=0.15$ and $d_{2}=0.5$ chosen so that each hemisphere spends a reasonable amount of time in each regime over the entire cycle (though with unrealistic annual variations in the background winds), regime correlations are plotted in Fig. 19 for varying average transmission coefficient $T_{0}$. Correlation values are plotted for both the full year and for boreal winters only $(0<$ $\nu t<\pi \bmod 2 \pi)$ when $T>0$ in (32). Also shown in the figure are correlations for a control run in which the ducts open and close according to (32), but with no annual cycle in thermal forcing [i.e., $d_{j}=0$ in (33)]. There are two principal conclusions to be drawn from the figure. First, the correlations (long-dashed line) for the model with an annual cycle in the ducts, but not in the thermal forcing, are comparable to correlations (dotted line) in the model with no annual cycle. Thus, partial synchronicity can be regained within one winter season after a period of decoupling. Synchronization with only occasional coupling has indeed been reported in another context (Morgul and Feki 1997). Second, the boreal winter correlations are seen to be enhanced slightly by the cycle in thermal forcing. The correlations (solid line) with an annual cycle in both ducts and forcing are higher almost everywhere than the correlations (dotted line) with no annual cycle, by an average of about one standard deviation in the latter (using the error bars shown in Fig. 13). Considered collectively, these differences appear to be significant.

While synchronized chaos has been reported in both directionally (Pecora and Carroll 1990; Rulkov et al. 


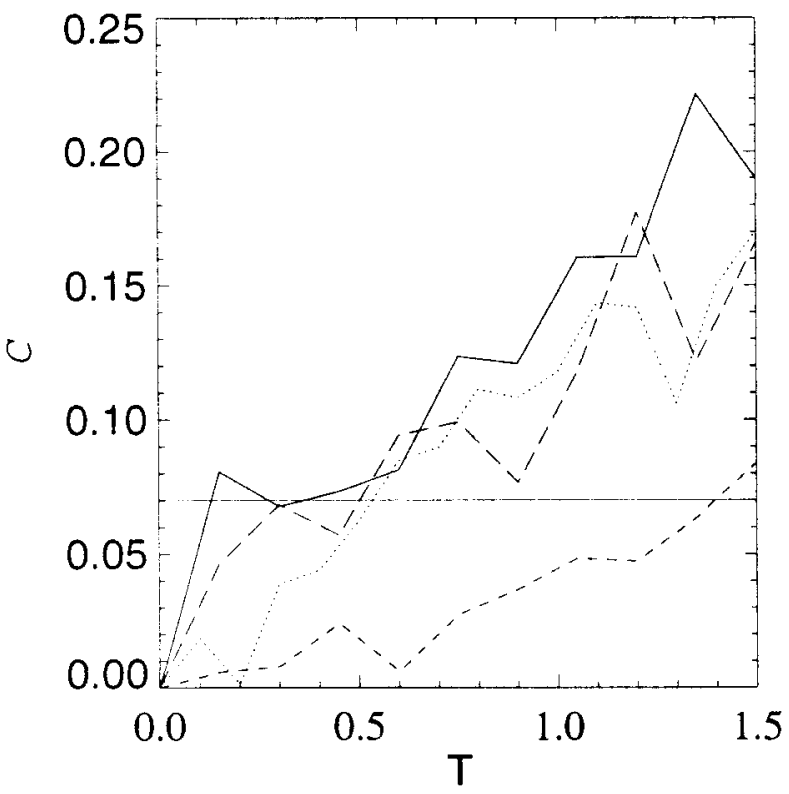

FIG. 19. The interhemispheric regime correlation $C$ vs parametric transmission coefficient as in Fig. 13 but with (a) an annual cycle in thermal forcing, given by (33), and an annual cycle in the ducts, given by (32), using statistics for boreal winters only (solid line); (b) an annual cycle in the ducts and steady forcing for boreal winters (long-dashed line); (c) no annual cycle, as in Fig. 13 (dotted line); (d) an annual cycle in both forcing and ducts, using full-year statistics (short-dashed line). The horizontal axis in (a) and (c) represents the average transmission coefficient $T_{0}$ in (32).

1995) and bidirectionally (Afraimovich et al. 1986; Fujisaka and Yamada 1983) coupled systems, the case of directional coupling seems more tractable analytically and appears to have been more widely studied for nonidentical systems (Rulkov et al. 1995). It is not known what can be said generally about the extent to which directionality actually aids synchronization or partial synchronization, but directionality does appear to play such a role here. That is, it is suggested that forcing one of two coupled systems more than the other tends to place that system in the role of the driver. The combined effects of the annual cycles in thermal forcing and in the ducts themselves lead to dominance by the Northern Hemisphere, which is more strongly forced by the higher equator-to-pole temperature gradient in boreal winter, when the ducts are open. The directional effect is evident in the plot of lagged correlations in Fig. 20 (though the largest correlations are simultaneous) and perhaps also explains the slight asymmetry with respect to time ordering in the lagged correlations in observations (Fig. 6).

If one believes that the truncations to wavenumber 2 capture the dynamics of each hemisphere, it is certainly plausible that the interhemispheric correlations would persist at higher orders of truncation since higher modes in a given hemisphere should have no greater effect on the opposite hemisphere than they have locally. This hypothesis is also supported by the falloff of the factors $\omega_{j} / \lambda_{j}$ in the coupling terms in (27) with increasing wavenumber

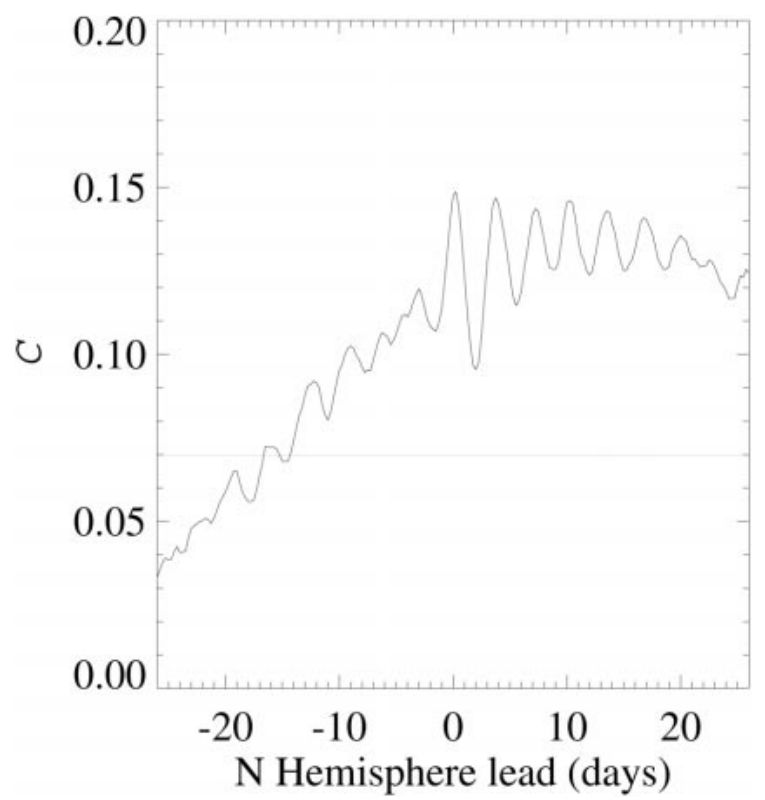

FIG. 20. Lagged correlation values $\mathcal{C}$, as in Fig. 6 but for a simulation of the two-hemisphere model with an annual cycle given by (32) and (33), over $5 \times 10^{4}$ nondimensional time units, considering only boreal winter periods. The horizontal axis specifies Northern Hemisphere lead in nondimensional time units, so that the range of dimensional lead times plotted is the same as that in Fig. 6. (Negative values represent Southern Hemisphere lead.) The thin line denotes the minimum significant correlation, and other parameters are as in Fig. 13.

and by the falloff of the nondiagonal coupling coefficients $W_{j k}(j \neq k)$ defined in (26) for fixed duct size as argued in detail in Duane (1997). Furthermore, synchronized chaos is known to be robust against significant noise in the coupling channel (Brown et al. 1994a,b).

Nevertheless, for physical authenticity, we examine interhemispheric correlations in a $K=3$ truncation in (27) of the model constructed from the barotropic vorticity equation (8). The illustration in Fig. 7 is actually for a planetary wavenumber 3 initial disturbance. A trajectory of the single-hemisphere, wavenumber 3 model, for a particular choice of forcing parameters, is shown in Fig. 21. It differs from the wavenumber 2 trajectories, such as the one in Fig. 9, principally in that the regimes in the $K=3$ case are less distinct, with a shorter regime residence time. Comparing to a typical regime history for observed data in the Northern Hemisphere, as shown in Fig. 22 for the NCEP-NCAR dataset, it is seen that regime-transition rates in reality lie somewhere between the wavenumber 2 and wavenumber 3 cases presented here and are closer to the latter. A blocked flow configuration for the two-hemisphere, wavenumber 3 model is depicted in Fig. 23. Correlations are plotted in Fig. 24 for a number of runs of the wavenumber 3 model with different initial conditions and different values of $T$. (An arbitrary threshold value $x_{1}=3$ was used to arbitrarily define regimes that may or may not correspond to dynamical regimes. In any case, the correlations were found to be rather insensitive to the choice of threshold value.) 


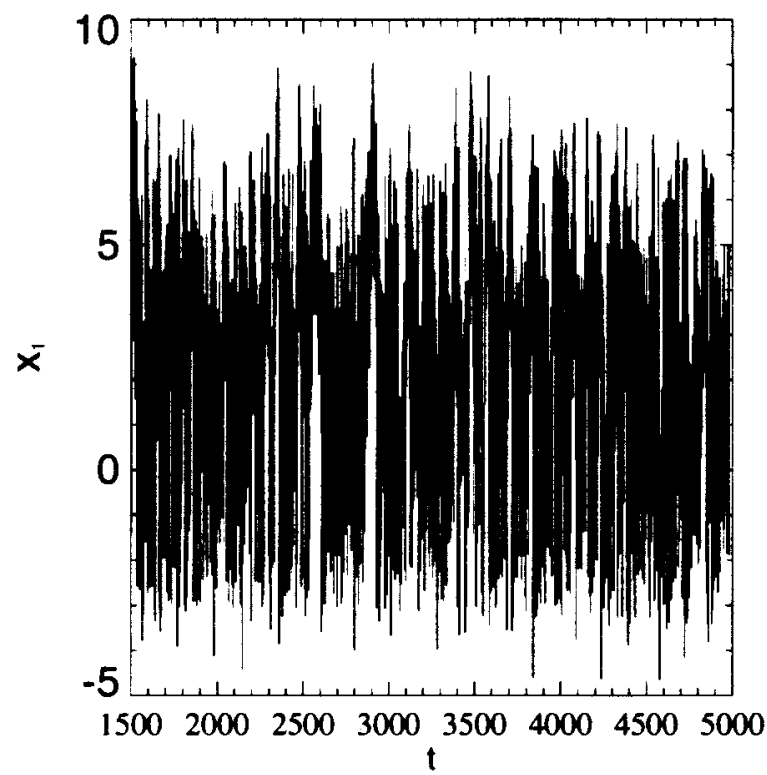

FIG. 21. Single-hemisphere trajectory as in Fig. 9 but for a model that includes modes up to wavenumber 3, i.e., for (14) with the summations over $\left|j_{1}\right|, j_{2} \leq 3$. Parameters are as in Fig. 9, except for the forcing terms $x_{1}^{*}=16, x_{11}^{*}=-15$, where $x_{11}^{*} \equiv \psi_{03}^{*} / b$.

The values of $C$ are not directly comparable to the wavenumber 2 values since the single-hemisphere forcing parameters in the two different truncations were chosen differently. The data in Fig. 24 considered as a whole, however, indicate that the interhemispheric correlations in the wavenumber 3 model are significant.

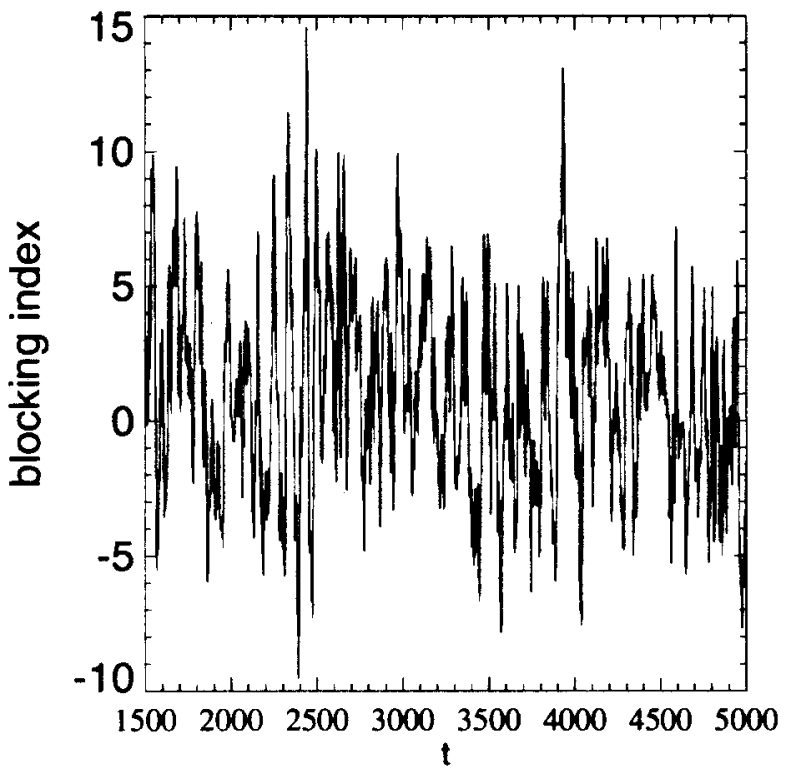

FIG. 22. Regime history of the Northern Hemisphere in NCEPNCAR data, over a time interval equal to that used in the model run in Fig. 21. The blocking index is defined as $\max _{\lambda, \Delta}\{\min [\mathrm{GHG} 1,(-10$ - GHG2)]\}, which is positive if and only if there is blocking somewhere in the hemisphere, according to the criterion in (4). [GHG1 and GHG2 are defined in (1).]

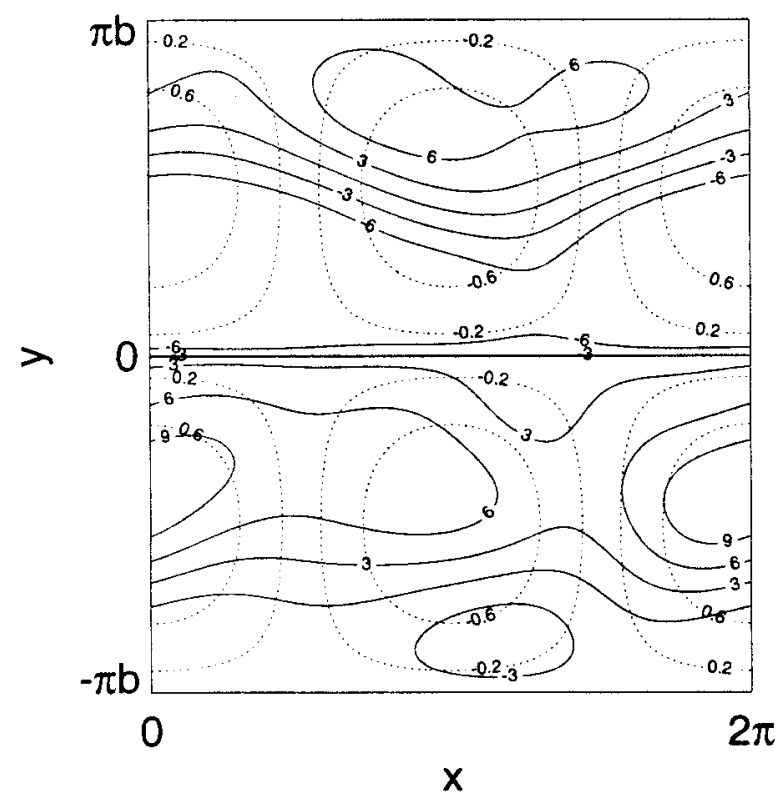

FIG. 23. Streamfunction and topographic height as in Fig. 14 but for a wavenumber 3 truncation of the two-hemisphere model at an instant when both hemispheres are blocked. Parameters are as in Fig. 14, except for increased forcing as in Fig. 21.

\section{Concluding remarks}

In summary, we have detected correlations between blocking events in the two hemispheres in observed data and have described a model in which blocking is the result of deterministic low-order chaos in each hemi-

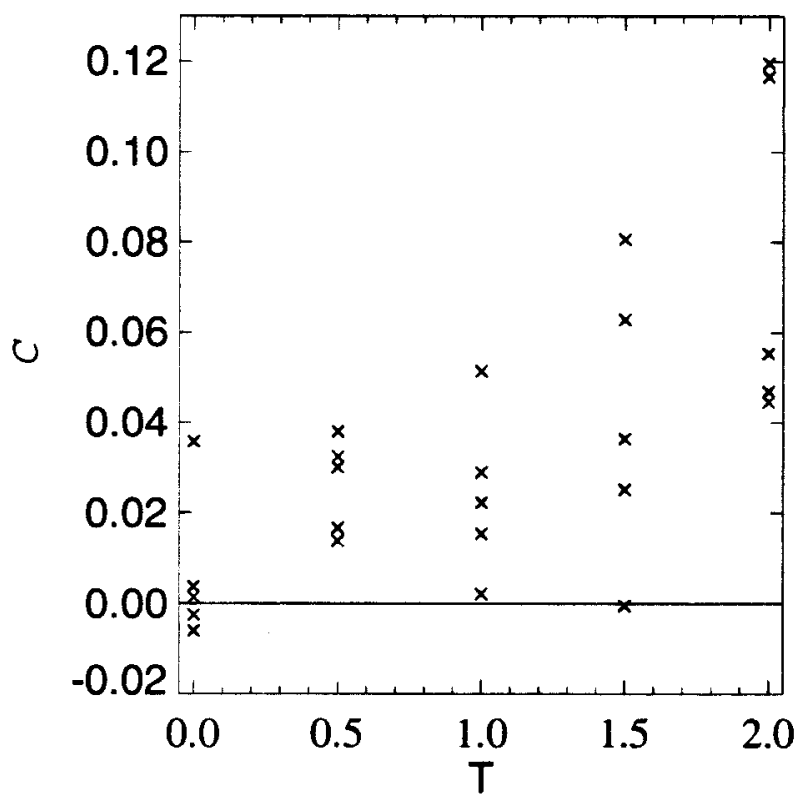

FIG. 24. The interhemispheric regime correlation $C$ as in Fig. 13 for various runs of the wave number $K=3$ model as depicted in Fig. 21, for simulations over 5000 nondimensional time units, but for different randomly chosen initial conditions and the indicated values of $T$. 
sphere, and hence is unpredictable, but still tends to occur simultaneously in the two hemispheres when they are weakly coupled. Both the observed and modeled correlations are small. For convenience, we have chosen to demonstrate interhemispheric correlations, which were described in Duane (1997) as partial synchronization, in a low-order truncation of a barotropic model. In the present paper, we showed that introducing an extended tropical region and topographic variations, including extra modes in the truncation, and decoupling the hemispheres half the time did not destroy the relationship between the two midlatitude flow systems, though some topographic configurations yielded anticorrelation instead of correlation. The annual cycle in thermal forcing enhanced the correlations slightly. It is hypothesized that including baroclinicity in an atmospheric system with the same approximate two-fold symmetry would leave the correlations intact. It is also believed that a two-hemisphere model based on spherical geometry that included enough modes to avoid the need for unrealistically large forcing, such as the model of Legras and Ghil (1985), would also exhibit partial synchronization. The single-hemisphere model we used in this study is descended from the sixcomponent model of Charney and DeVore. Those authors chose to study an oversimplified model because its multiple equilibria coincided qualitatively with those found in the real atmosphere, with the following caveat: "We regard our preliminary findings as useful primarily for their heuristic character, not for their detailed explanation of specific phenomena" (Charney and DeVore 1979). Given the robustness we have demonstrated in the modeled interhemispheric correlations, we regard our present findings as similiarly useful.

While we have shown that the interaction between the two midlatitude systems through a passive Tropics can perhaps explain the observed blocking cooccurrence statistics, one must consider the alternative explanation that blocking in both hemispheres is triggered by common tropical forcing. One can show that tropical variations on interannual timescales, such as variations in tropical Pacific sea surface temperatures, are not the cause of the interhemispheric correlations: if one computes the correlation for each winter in the dataset separately, so as to exclude low-frequency effects, the average of these values is only about $10 \%$ smaller than the correlation value for the dataset as a whole. This still leaves the possibility that tropical variability on synoptic timescales, rather than midlatitude synoptic variability, may explain the observed correlations. The two mechanisms are not exclusive of one another. A definitive way to assess the relative importance of the two mechanisms would be to examine the behavior of the interhemispheric correlations in a general circulation model, as the tropical region is artificially relaxed, to an increasing degree, to a climatological basic state.

There is indeed evidence that the interaction between the Tropics and the midlatitude systems is bidirectional. Rossby waves from the midlatitudes can stimulate con- vective activity in the Tropics (Kiladis and Weickmann 1992), for instance. But since tropical activity affects both hemispheres, any such impact on the Tropics provides a mechanism for effective interaction between the two midlatitude systems. [The effect on the summer hemisphere, though weak because of the stronger easterlies there (Webster 1982), is completely analogous to the effect of Rossby waves crossing into the summer hemisphere through a passive Tropics.] The combination of such second-order effects and the direct interaction mechanism at the longitudes of the ducts may give rise to partial synchronization similar to that found in our admittedly oversimplified model since the overall symmetry of the system is maintained with an active Tropics.

In the synchronized chaos view, the qualitative behavior of each component subsystem is determined by its intrinsic dynamics. Thus, the preferred location of blocking in each hemisphere is determined by that hemisphere's effective topography. Only the timing of the blocking events, which is unpredictable in a chaotic system and, thus, sensitive to external input, is affected by the opposite hemisphere. A weak signal across the hemisphere is still necessary, but such is provided by Rossby waves emanating from the westerly ducts and traversing a great circle across the entire hemisphere (Hoskins and Karoly 1981), either reinforcing or inhibiting the intrinsic hemisphere-wide flow patterns that give rise to blocking.

More generally, the climate system is full of semiautonomous, weakly interacting, individually chaotic subsystems to which the partially synchronized chaos paradigm may apply. Empirical evidence sometimes suggests correlations between the gross states of such subsystems, even when the subsystems are different. The tendency for strong monsoons to occur during La Niña years and weak monsoons during El Niño years (Yasunari 1990; Webster and Yang 1992) is one example. A tendency for the the Pacific-North American (PNA) and North Atlantic Oscillation (NAO) patterns to covary in some decades is another (Hurrell 1998, personal communication). The fact that the PNA-NAO correlations and the monsoon-ENSO correlations themselves vary on decadal timescales is possibly related to the on-off synchronization behavior that occurs in partially synchronized systems, such as our interhemispheric model, in a different parameter regime (Duane 1997). The task of constructing simplified models of the monsoon-ENSO or PNA-NAO interactions is more difficult than for the coupling that was considered in this paper. Models of the interacting systems formulated at a level higher than that of the primitive equations, however, may explain the observed correlations. That such correlations are to be expected regardless of the details of such models, however, is very much in keeping with the original ideas of Walker (1924), which preceded the later mechanistic views. The number of other measurable relationships that link remote parts of the earth's 
climate and are presently unappreciated may be considerable.

Acknowledgments. This work was supported by National Science Foundation Grants NSF-ATM-9526030 and NSF-ATM-9312760, and by NOAA Grant DOC NA56GP0230. One of us (GSD) would like to thank Dr. Sue Haupt for her role in his support. We thank Dr. Doug Nychka for useful discussions about the statistical significance of the correlations in the data and the model.

\section{APPENDIX}

\section{Coefficients in the Spectral Expansion of the Barotropic Vorticity Equation}

Nonvanishing values of the coefficients $c_{j l m}$ and $b_{j l}$ in the expansion (14) of the barotropic vorticity equation to arbitrary order:

$$
\lambda_{j} \dot{\psi}_{j}=\frac{1}{2} \sum_{l, m} c_{j l m}\left(\lambda_{l}-\lambda_{m}\right) \psi_{l} \psi_{m}+\gamma \sum_{l, m} c_{j l m} \psi_{l} h_{m}+\sum_{l} b_{j l} \psi_{l}-\mu \lambda_{j}\left(\psi_{j}-\psi_{j}^{*}\right),
$$

where $j=\left(j_{1}, j_{2}\right), l=\left(l_{1}, l_{2}\right)$, and $m=\left(m_{1}, m_{2}\right)$ are given below. Values of $c_{j l m}$ and $b_{j l}$ for combinations of subscripts not described below are zero: ${ }^{2}$

$$
\begin{aligned}
& j_{1}=0 ; \quad l_{1}=-m_{1} ; \quad j_{2}+l_{2}+m_{2} \text { odd } \quad c_{j l m}=\frac{i \sqrt{2} l_{1}}{\pi b}\left\{\left(l_{2}+m_{2}\right)\left[\frac{1}{j_{2}+l_{2}+m_{2}}-\frac{1}{j_{2}-l_{2}-m_{2}}\right]\right. \\
& \left.-\left(l_{2}-m_{2}\right)\left[\frac{1}{j_{2}+l_{2}-m_{2}}-\frac{1}{j_{2}-l_{2}+m_{2}}\right]\right\} \\
& l_{1}=0 ; \quad j_{1}=m_{1} ; \quad j_{2}+l_{2}+m_{2} \text { odd } \quad c_{j l m}=\frac{i \sqrt{2} j_{1}}{\pi b}\left\{\left(m_{2}+j_{2}\right)\left[\frac{1}{l_{2}+m_{2}+j_{2}}-\frac{1}{l_{2}-m_{2}-j_{2}}\right]\right. \\
& \left.-\left(m_{2}-j_{2}\right)\left[\frac{1}{l_{2}+m_{2}-j_{2}}-\frac{1}{l_{2}-m_{2}+j_{2}}\right]\right\} \\
& m_{1}=0 ; \quad j_{1}=l_{1} ; \quad j_{2}+l_{2}+m_{2} \text { odd } \quad c_{j l m}=-\frac{i \sqrt{2} l_{1}}{\pi b}\left\{\left(j_{2}+l_{2}\right)\left[\frac{1}{m_{2}+j_{2}+l_{2}}-\frac{1}{m_{2}-j_{2}-l_{2}}\right]\right. \\
& \left.-\left(j_{2}-l_{2}\right)\left[\frac{1}{m_{2}+j_{2}-l_{2}}-\frac{1}{m_{2}-j_{2}+l_{2}}\right]\right\} \\
& j_{1}=l_{1}+m_{1} ; \quad j_{2}=l_{2}+m_{2} \quad c_{j l m}=\frac{i}{b \sqrt{2}}\left(l_{1} m_{2}-l_{2} m_{1}\right) \\
& j_{1}=l_{1}+m_{1} ; \quad j_{2}=l_{2}-m_{2} \quad c_{j l m}=\frac{i}{b \sqrt{2}}\left(l_{1} m_{2}+l_{2} m_{1}\right) \\
& j_{1}=l_{1}+m_{1} ; \quad j_{2}=-l_{2}+m_{2} \quad c_{j l m}=-\frac{i}{b \sqrt{2}}\left(l_{1} m_{2}+l_{2} m_{1}\right) \\
& j_{1}=-l_{1} ; \quad j_{2}=l_{2} \quad b_{j l}=i \beta l_{1}
\end{aligned}
$$

\section{REFERENCES}

Afraimovich, V. S., N. N. Verichev, and M. I. Rabinovich, 1986: Stochastic synchronization of oscillation in dissipative systems. Radiophys. Quantum Electron., 29, 795-803.

\footnotetext{
${ }^{2}$ Our coefficients differ from those given by the general expressions in de Swart $(1988,1989)$, mainly because the symmetry relation $c_{i l m}=c_{l m j}$ used in that work does not hold when the basis functions $\Phi_{j}$ are complex. Our coefficients do agree with those used in de Swart's wavenumber 2 model.
}

Ashwin, P., J. Buescu, and I. Stewart, 1994: Bubbling of attractors and synchronisation of chaotic oscillators. Phys. Lett. A, 193, 126-139.

Bjerknes, J., 1969: Atmospheric teleconnections from the equatorial Pacific. Mon. Wea. Rev., 97, 163-172.

Brown, R., N. F. Rulkov, and N. B. Tufillaro, 1994a: The effects of additive noise and drift in the dynamics of the driving on chaotic synchronization. Phys. Lett. A, 196, 201-205.

,-- , and — 1994b: Synchronization of chaotic systems: The effects of additive noise and drift in the dynamics of the driving. Phys. Rev. E, 50, 4488-4508.

Charney, J. G., and J. G. DeVore, 1979: Multiple flow equilibria in the atmosphere and blocking. J. Atmos. Sci., 36, 1205-1216. 
Coughlan, M. J., 1983: A comparative climatology of blocking action in the two hemispheres. Aust. Meteor. Mag., 31, 3-13.

de Swart, H. E., 1988: Low-order spectral models of the atmospheric circulation: A survey. Acta Appl. Math., 11, 49-96.

- 1989: CWI Tract 60: Vacillation and Predictability Properties of Low-Order Atmospheric Spectral Models. Stichting Mathematisch Centrum, $121 \mathrm{pp}$.

Duane, G. S., 1997: Synchronized chaos in extended systems and meteorological teleconnections. Phys. Rev. E, 56, 6475-6493.

Fujisaka, H., and T. Yamada, 1983: Stability theory of synchronized motion in coupled-oscillator systems. Prog. Theor. Phys., 69, 32-47.

Hoskins, B. J., and D. J. Karoly, 1981: The steady linear response of a spherical atmosphere to thermal and orographic forcing. $J$. Atmos. Sci., 38, 1179-1196.

Jin, F.-F., J. D. Neelin, and M. Ghil, 1994: ENSO on the devil's staircase. Science, 264, 70-72.

Kalnay, E., and Coauthors, 1996: The NCEP/NCAR 40-year reanalysis project. Bull. Amer. Meteor. Soc., 77, 437-471.

Kiladis, G. N., and K. M. Weickmann, 1992: Extratropical forcing of tropical Pacific convection during northern winter. Mon. Wea. Rev., 120, 1924-1938.

Kocarev, L., Z. Tasev, and U. Parlitz, 1997: Synchronizing spatiotemporal chaos of partial differential equations. Phys. Rev. Lett. 79, 51-54.

Legras, B., and M. Ghil, 1985: Persistent anomalies, blocking and variations in atmospheric predictability. J. Atmos. Sci., 42, 433471.

Lorenz, E. N., 1991: Dimension of weather and climate attractors Nature, 353, 241-244.

Morgul, O., and M. Feki, 1997: Synchronization of chaotic systems by using occasional coupling. Phys. Rev. E, 55, 5004-5010.

Morse, P. M., and H. Feshbach, 1953: Green functions. Methods of Theoretical Physics, McGraw-Hill, 795-803.

Palmer, T. N., 1993: Extended-range atmospheric prediction and the Lorenz model. Bull. Amer. Meteor. Soc., 74, 49-65.

Pecora, L. M., and T. L. Carroll, 1990: Synchronization in chaotic systems. Phys. Rev. Lett., 64, 821-824.

Rex, D. F., 1950: Blocking action in the middle troposphere and its effect upon regional climate. Part I: An aerological study of blocking action. Tellus, 2, 196-211.

Rulkov, N. F., M. M. Sushchik, and L. S. Tsimring, 1995: Generalized synchronization of chaos in directionally coupled chaotic systems. Phys. Rev. E, 51, 980-994.

Sushchik, M. M., 1996: Synchronized chaotic oscillations. Ph. D. dissertation, University of California, San Diego, 174 pp. [Avail- able from University of California at San Diego, La Jolla, CA 92093.]

Tibaldi, S., and F. Molteni, 1990: On the operational predictability of blocking. Tellus, 42A, 343-365.

Treidl, R. A., E. C. Birch, and P. Sajecki, 1981: Blocking action in the Northern Hemisphere: A climatological study. Atmos.Ocean, 19, 1-23.

Tziperman, E., M. A. Cane, and S. Zebiak, 1995: Irregularity and locking to the seasonal cycle in an ENSO prediction model as explained by the quasi-periodicity route to chaos. J. Atmos. Sci., 52, 293-306.

, H. Scher, S. E. Zebiak, and M. A. Cane, 1997: Controlling spatiotemporal chaos in a realistic El Niño prediction model. Phys. Rev. Lett., 79, 1034-1037.

van Loon, H., 1956: Blocking action in the Southern Hemisphere Pt. 1. Notos, 5, 171-178.

Venkataramani, S. C., B. R. Hunt, and E. Ott, 1996: Bubbling transition. Phys. Rev. E, 54, 1346-1360.

Walker, G. T., 1924: Correlation in seasonal variations of weather: A further study of world weather. Memoirs of the India Meteorological Department, India Meteorological Dept., 275-332.

Webster, P. J., 1981: Mechanisms determining the atmospheric response to sea surface temperature anomalies. J. Atmos. Sci., 38, $554-571$.

, 1982: Seasonality in the local and remote atmospheric response to sea surface temperature anomalies. J. Atmos. Sci., 39, 41-52. , and J. L. Keller, 1974: Strong long-period tropospheric and stratospheric rhythm in the Southern Hemisphere. Nature, 248, $212-213$.

, and J. R. Holton, 1982: Cross-equatorial response to middlelatitude forcing in a zonally varying basic state. J. Atmos. Sci., 39, 722-733.

systems. Quart. J. Roy. Meteor. Soc., 118, 877-926.

- , and H.-R. Chang, 1998: Atmospheric wave propagation in heterogeneous flow: Basic flow controls on tropical-extratropical interaction and equatorial wave modification. Dyn. Atmos. Oceans, 27, 91-134.

Wright, A. D. F., 1974: Blocking action in the Australian region. Australian Bureau Meteorology Tech. Rep. 10, 29 pp. [Available from Bureau of Meteorology, G.P.O. Box 1289K, Melbourne, VIC 3001, Australia.]

Yasunari, T., 1990: Impact of Indian monsoon on the coupled atmosphere/ocean system in the tropical Pacific. Meteor. Atmos. Phys., 44, 29-41.

Zebiak, S. E., and M. A. Cane, 1987: A model El Niño-Southern Oscillation. Mon. Wea. Rev., 115, 2262-2278. 